\title{
Performance analysis of relay selection in cooperative networks over Rayleigh flat fading channels
}

\author{
Hanan Al-Tous and Imad Barhumi
}

\begin{abstract}
Performance analysis of an up-link cooperative diversity system is investigated; sharing of the two ordered best relays over Rayleigh flat fading channel is introduced to establish full diversity order for both users. The two users are competing for the same best relay, so assigning the best relay for one user, and the next-best relay for the other shows different diversity orders. The relays are ordered based on the end-to-end signal to noise ratio (SNR) of the source-relay-destination links. In this sense, relay selection is examined under different criteria. Mainly, the ordered best relay, the ordered next-best relay, and equally sharing the two best ordered relays. To this end, analytical expression, for the moment generating function (MGF) is derived, and used to find the probability density function (PDF), and the cumulative density function of the end-to-end SNR for decode and forward (DF) sharing scenario. Furthermore, the MGF of the upper bound of the end-to-end SNR for amplify and forward (AF) sharing scenario is also derived. Sharing the two ordered best relays shows better performance in the bit error probability (BEP) than using the next-best relay alone in DF relay systems, while exploiting the full diversity of the system. Sharing of the two ordered best relays in AF relay systems shows better BEP performance than using the best relay alone. Distributed space time block coding and distributed beamforming (BF) scenarios at the relays that utilize the bandwidth more efficiently are also explored. It is found that the BEP performance of the two ordered best relays distributed BF with equal power allocation for AF (at high SNR) and DF (for all SNRs) schemes outperforms the BEP performance of the best ordered relay alone. The BEP performance for the DF distributed BF scheme with equal power allocation approaches the BEP performance of the optimum power assignment under global power sum constraint. Numerical simulations are used to validate the analysis.
\end{abstract}

Keywords: Cooperative communications, Amplify and forward, Decode and forward, Relay selection, Distributed beamforming, Distributed space time block coding, Bit error probability, Outage probability, Diversity order

\section{Introduction}

Cooperative wireless networks have been adopted in order to address the requested increase in capacity and to improve the wireless link performance. In cooperative communication, multiple relays are utilized to provide reliable, high data rate, and efficient communication. Recent activities on cooperative communication mostly stem from the potential of wireless applications and are motivated by many recent articles [1-4]. In most practical cooperative diversity protocols, transmission is accomplished in two phases: a broadcast phase and a

*Correspondence: imad.barhumi@uaeu.ac.ae

College of Engineering, UAE University, Al Ain, UAE multi-access phase. In the broadcast phase, the source node broadcasts its message to the assisting relays and to the destination node. Whereas in the second phase, the relays collaboratively transmit the received information to the destination node. Two relaying schemes are generally used for cooperative diversity networks: amplifyand-forward (AF) and decode-and-forward (DF). In AF, the received noisy message is amplified and forwarded to the destination node. The destination node combines the information sent by the user (source) and the partner relay(s), and makes a final decision on the transmitted symbol. Although noise is amplified by cooperation, the base station receives two or more independently faded

\section{贷 Springer}

(c) 2012 Al-Tous and Barhumi; licensee Springer. This is an Open Access article distributed under the terms of the Creative Commons Attribution License (http://creativecommons.org/licenses/by/2.0), which permits unrestricted use, distribution, and reproduction in any medium, provided the original work is properly cited. 
versions of the signal and can make better decision on the detection of the transmitted information. In DF, the received noisy message at the relay is decoded first, and then the relay re-encodes the decoded message, and forwards it to the destination node. In the case of accurate symbol estimation, DF outperforms AF relaying, where the relay can reliably decode the source node message as the noise will not be amplified [5-8]. Diversity order two is achieved at high signal to noise ratio (SNR) using one relay for $\mathrm{AF}$ and adaptive DF schemes, using maximum ratio combining (MRC) technique to demodulate the received signals. In adaptive DF, the relay transmits if it decodes the message correctly (e.g., by using cyclic redundancy check), or otherwise keeps silent $[9,10]$. In common cooperative diversity networks with $N$ relaying nodes, $N+1$ orthogonal channels or time slots are used to provide $N+1$ diversity order, which encounters bandwidth penalty [11-14]. In [15], opportunistic best relay selection is used to utilize the resources efficiently; only two channels or time slots are required despite the number of relays, while maintaining a full diversity order $N+1$, when the best relay is only used. Laneman et al. [3] proved that best relay selection achieves full diversity order for AF and DF cooperation schemes under flat fading Rayleigh channels. In there, the performance was measured in terms of the outage events and the associated outage probabilities. Different criteria for relay selection are investigated with their achievable diversity order in [16]. The average symbol error probability (SEP) of the cooperative system is used to analyze the performance of various systems and channel models as in [8].

In [17], Ikki and Ahmed investigated the $k$ th ordered best relay and proved that the diversity order increases with the number of relays and decreases linearly with the order of the relay, i.e., the best ordered relay is denoted as $k=0$ that achieves full diversity order $N+1$, whereas the next-best ordered relay is denoted as $k=1$ which achieves a diversity order $N$. Closed form expressions for the error and outage probabilities over identical and non-identical Rayleigh fading channels were derived. Single and multiple relay selection schemes were investigated. Several SNR sub-optimum multiple relay selection schemes with linear complexity in the number of relays were used [16]. Multiple potential relays and multiple simultaneous transmissions were introduced; where each source pairs with a single best relay, the outage probability of the proposed scheme is derived in [18]. In [19], joint selection scheme in multi-source multi-relay networks was considered, by selecting the best source node and the best relay to access the channel. Diversity of multiuser two-hop cooperative relay network was developed for different relaying protocols and tight closed-form expressions for the outage probability and the SEP were derived [20].
In this article, we consider the following scenario. A selected best relay of one user is the same best relay for another user in a power limited relay system. The probability of two users competing for the same relay is significant and comparable to the probability of having different best relays for a multi-user system with similar average channel conditions as proved in [18]. This case becomes visible in multi-user scenarios, or multi-carrier systems like OFDMA. In this sense, two solutions were proposed in literature; the first solution is to use the best relay for user one, and the next-best relay for the other user. So, it is transformed to the $k$ th best ordered relay selection problem, which was investigated in $[17,21-23]$, by this solution the users achieve different diversity orders. The second solution is to use the best relay for the two users with half power for each user if this choice gives better performance than using the next-best relay alone with full power, which was proposed in [18]. However, no analytical results for the SEP, or outage probability have been derived because of the mathematical complexity. In summary, the two solutions stand on using only one relay for each user.

In here, we propose a different scheme, in which the best and the next-best relays are equally shared between the two users/carriers. The relays are ordered based on the instantaneous end-to-end SNR of the source-relaydestination links. Each user will have the chance to use the best and the next-best ordered relays in a predetermined manner. Equal opportunity of using the best relay is the fairground to build up this scheme. This solution is motivated by the observation that the bit error probability (BEP) performance of the two best order relays AF systems with half power for each relay in a three-time slots scenario outperforms the best relay BEP performance. The three-time-slots are used as follows: the first-timeslot is used to transmit the sources' data to the relays and the destination, the second-time-slot is used to relay the processed data from the best relay to the destination, and the third-time-slot is used to relay the processed data from the next-best relay to the destination. In addition, sharing the two ordered best relays for both $\mathrm{AF}$ and DF cooperative schemes for independent identically flat fading Rayleigh channels, utilizing two-time slots using distributed space time block coding (STBC) or distributed beamforming (BF) are also investigated to exploit the channel efficiently. The novelty of this work, is that relays are selected to attain full diversity order for both users. Our scheme places full diversity selection at the core of the design scheme, and takes into consideration the limited available power at the relay. The available power at the relay is used to support the two users, which is equally split between the two users.

The moment generating function (MGF) formula of the received SNR of the two ordered best relays (the best, 
and the next-best) after using MRC is derived assuming equal power sharing in which each relay transmits with half power. Optimal power allocation for the two users could also be used, however, the problem is not analytically tractable for AF. Equal power allocation for orthogonal sharing DF is a fair allocation that maximizes the minimum end-to-end SNR of the two users over the source-relay-destination links. Exact expressions for the $\mathrm{BEP}$, and the outage probability are derived based on the MGF, which can also be used for performance evaluation or comparison purposes for the following cases: orthogonal DF, STBC-DF, and BF-DF. Upper and lower bounds of the end-to-end SNR for orthogonal AF, and BF-AF are also derived. The BEP performance of sharing the two ordered best relays is compared with the BEP performance of the best, and the next-best ordered relays using simulations.

The remaining of the article is organized as follows. Section "System and channel model" presents the system model. Analytical expressions of the bit error and outage probabilities for the three-time slots scenarios are derived in Section "Scenario one (orthogonal three-time slots scenario)". Analytical expressions of the BEP for the two-time slots scenarios are derived in Section "Scenario two (two-time slots scenario)". Numerical results and conclusions are presented in Section "Numerical results and discussion" and Section "Conclusions", respectively.

\section{System and channel model}

Communications between two sender nodes (sources $S_{1}$ and $S_{2}$ ) and a common destination terminal $(D)$ over a Rayleigh flat fading channels are facilitated by sharing the two ordered best relay stations $R_{b 0}$ and $R_{b 1}$, from $N$ available relay nodes for AF scheme, and from a set $C$ of relay nodes that can decode the sources' messages correctly for DF scheme. These scenarios are explained next. The system under consideration is depicted in Figure 1. In this model, the channel characterizing the link between source $S_{j}$ and relay $R_{l}$ is denoted as $h_{S_{j} R_{l}}$, and the channel characterizing the link between relay $R_{l}$ and destination node $D$ is denoted as $h_{R_{l} D}$. Moreover, the channel identifying the link between source $S_{j}$ and destination node $D$ is denoted as $h_{S_{j} D}$. The channels $h_{S_{j} R_{l}}, h_{R_{l} D}$, and $h_{S_{j} D}$ are assumed independent identically distributed (iid) Rayleigh random variables for $l \in\{1, \ldots, N\}$ and $j \in\{1,2\}$. The received noise at all links is assumed to be iid additive white Gaussian noise (AWGN) with zero mean and variance $N_{0} / 2$ per dimension.

For relay selection schemes, the channel characterizing the link between the source $S_{j}$ and the $i$ th ordered best relay $R_{b i}$ is denoted as $h_{S_{j} R}^{(i)}$, and the channel characterizing the link between the $i$ th ordered best relay and the

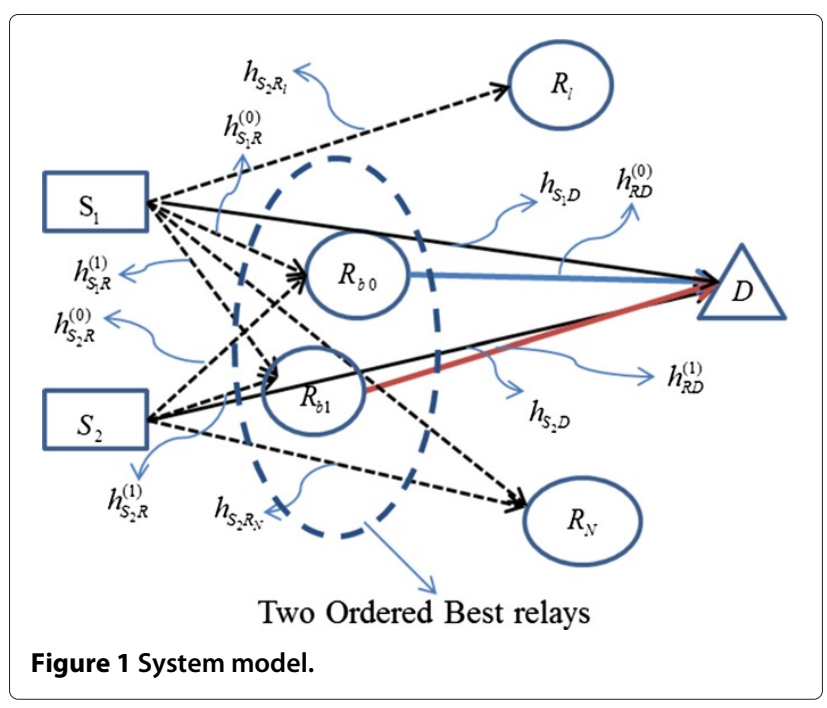

destination is denoted as $h_{\mathrm{RD}}^{(i)}$, where $i \in\{0,1\}$. In all scenarios, sources and relays have the same power capability. In relay selection, we assume that the best relay is the same for the two users, but the next-best relay may not be the same. However, this will not affect the analysis. For distributed STBC scenario, the best and the next-best relays are assumed to be the same for the two users in order to implement this scenario.

In this article, time division multiple access (TDMA) is considered. For a two-users case, each time slot is divided into two time sub-slots. Frequency division multiple access (FDMA) can also be considered in a similar fashion, where each frequency sub-band in FDMA corresponds to a time sub-slot in TDMA. The sharing scenarios are classified into two categories: three-time slots scenario (orthogonal), and two-time slots scenario (non-orthogonal). In both categories, the first-time slot is used for the sources' transmission to the destination node and the relay nodes (broadcast phase). Orthogonal here refers to relay transmission, where the best relay transmits in the second-time slot, and the next-best relay transmits in the third-time slot (no interference). The second and third time slots are subdivided into $T_{k 1}$, and $T_{k 2}$ for $k \in\{2,3\}$ to transmit user's 1 and 2 data, respectively. For two-time slots scenario, distributed STBC or distributed BF transmission schemes are used to relay the data from the two best ordered relays to the destination node for the two users simultaneously. Non-orthogonal here refers to the transmission in the second-time slot; the best, and the next-best relays transmit at the same time. Sub-slot $T_{2 j}$ is used by the best and next-best relays for transmitting user's $j$ data simultaneously for BF scenarios. The instantaneous value of the phase of the channel state information (CSI) of the source-relay and the relaydestination links are required to be available at the best, 
and the next-best relays to perform distributed BF. For distributed STBC the best and next-best relays transmit the re-encoded signal or the complex conjugate of the the re-encoded signal in a predetermined way as will be explained in Section "DF with distributed STBC (STBC-DF)". The destination node for all scenarios, combines the directed and the relayed signals using MRC, where the received signals from all independent paths are co-phased, weighted, and combined, assuming the destination knows the instantaneous CSI from the sources, and the relays.

The instantaneous CSI is kept invariant over multiple transmission intervals. So, the selection of the best, and the next-best relays is performed once for multiple transmissions. Relay selection is performed before data transmission. The best relay and next-best relay can be determined for both AF and DF in a centralized or distributed fashion, depending on where the decision is carried out. In centralized relay selection, the destination node based on the end-to-end SNR of the source-relay-destination links, determines the best, and the next-best relays, and informs the selected relays through feedback channels. In distributed relay selection, each relay acquires the instantaneous CSI of the two links (relay-destination, and source-relay), the CSI of the relay-destination link can be acquired by allowing the destination to transmit a pilot signal. The relay then can determine the CSI of the relay-destination link assuming that the relay-destination link is symmetric. Besides, the CSI of the source-relay link can be determined at the relay from the source request to transmit. Based on CSI of the two links (relaydestination, and source-relay), the relay sets a timer and remains silent inversely proportional to the end-to-end SNR. The relay whose timer expires first or second will broadcast a signal to other relays, indicating that they can go to a sleep mode for the rest of the current transmission period. If the relay receives two signals from other relays before its timer goes to zero, it can go to a sleep mode, otherwise, it will be the best relay or the next-best relay $[15,24]$.

Scenario one (orthogonal three-time slots scenario) In this scenario, three-time slots are used. The selected relays are shared between the two sources (users). The sharing is in terms of the relays' transmitted power. As illustrated in Table 1, where $T_{x_{j}}$ stands for transmission of user $j$ (source $S_{j}$ ) data $x_{j}$, and $R_{x_{j}}$ stands for receiving user $j$ (source $S_{j}$ ) data $x_{j}$. The received signal $y_{T_{1 j}}^{(D)}$ at the destination node $D$, in time slot $T_{1}$ is obtained as follows:

$$
y_{T_{1 j}}^{(D)}=\sqrt{P_{S}} h_{S_{j} D} x_{j}+n_{1 j}^{(D)} \quad j=1,2 .
$$

Table 1 Three-time slots scenario

\begin{tabular}{lllllll}
\hline & $T_{\mathbf{1 1}}$ & $T_{\mathbf{1 2}}$ & $T_{\mathbf{2 1}}$ & $\boldsymbol{T}_{\mathbf{2 2}}$ & $\boldsymbol{T}_{\mathbf{3 1}}$ & $\boldsymbol{T}_{\mathbf{3 2}}$ \\
\cline { 2 - 7 }$S_{1}$ & $T_{x_{1}}$ & - & - & - & - & - \\
$S_{2}$ & - & $T_{x_{2}}$ & - & - & - & - \\
$R_{b 0}$ & $R_{x_{1}}$ & $R_{x_{2}}$ & $T_{x_{1}}$ & $T_{x_{2}}$ & - & - \\
$R_{b 1}$ & $R_{x_{1}}$ & $R_{x_{2}}$ & - & - & $T_{x_{1}}$ & $T_{x_{2}}$ \\
$D$ & $R_{x_{1}}$ & $R_{x_{2}}$ & $R_{x_{1}}$ & $R_{x_{2}}$ & $R_{x_{1}}$ & $R_{x_{2}}$ \\
\hline
\end{tabular}

The received signal $y_{T_{1 j}}^{\left(R_{l}\right)}$ at the relay node $R_{l}$, in time slot $T_{1}$ is obtained as:

$$
y_{T_{1 j}}^{\left(R_{l}\right)}=\sqrt{P_{s}} h_{S_{j} R_{l}} x_{j}+n_{1 j}^{\left(R_{l}\right)} \quad j=1,2 \& l=1, \ldots, N .
$$

In particular, the received signal at the ordered best relays $R_{b 0}$ and $R_{b 1}$ in time slot $T_{1}$ is obtained as:

$$
y_{T_{1 j}}^{\left(R_{b i}\right)}=\sqrt{P_{s}} h_{S_{j} R}^{(i)} x_{j}+n_{1 j}^{\left(R_{b i}\right)} \quad j=1,2 \& i=0,1
$$

where $n_{1 j}^{(D)}, n_{1 j}^{\left(R_{l}\right)}$, and $n_{1 j}^{\left(R_{b i}\right)}$ are the additive noise at the destination $D$, at the the relays $R_{l}$, and at the best ordered relays $R_{b i}$, respectively. The relays are ordered based on the end-to-end SNR of the source-relay-destination links as explained for DF as well as for AF scenarios in Sections "DF orthogonal three-time slots scenario" and "AF orthogonal three-time slots scenario", respectively. The transmitted symbol $x_{j}$ is drawn from a constellation with unit energy, $P_{S}$ is the source transmitted power. The instantaneous received SNR at the destination node from the source $S_{j}$ through the direct link over Rayleigh flat fading channel is defined as $\gamma_{S_{j} D}$. It is computed using (1) as $\gamma_{S_{j} D}=\gamma_{0}\left|h_{S_{j} D}\right|^{2}$, where $\gamma_{0}=\frac{P_{S}}{N_{0}} \cdot \gamma_{S_{j} D}$ is a random variable exponentially distributed with parameter $\lambda_{S_{j} D}$. To simplify the forthcoming analysis, the source-destination links of users 1 and 2 are assumed iid, i.e., $\lambda_{S_{1} D}=\lambda_{S_{2} D}=$ $\lambda_{\mathrm{SD}}=\frac{1}{\gamma_{0} E\left\{\left|h_{S_{j} D}\right|^{2}\right\}}$, where $E\{\cdot\}$ stands for statistical expectation. Similarly, the instantaneous received SNR at the relay $R_{l}$ from the $j$ th source is computed using (2) as $\gamma_{S_{j} R_{l}}=$ $\gamma_{0}\left|h_{S_{j} R_{l}}\right|^{2}$, where $\gamma_{S_{j} R_{l}}$ is also exponentially distributed random variable with parameter $\lambda_{S_{j} R_{l}}$. The source relay links are assumed to have the same average $\lambda_{S_{j} R_{l}}=\lambda_{\mathrm{SR}}=$ $\frac{1}{\gamma_{0} E\left\{\left|h_{S_{j} R_{l}}\right|^{2}\right\}}, \forall l \in\{1, \ldots, N\}$, and $\forall j \in\{1,2\}$. For an exponentially distributed random variable $X$ with parameter $\lambda$, the mean is given as $\mu_{X}=E\{X\}=\frac{1}{\lambda}$.

The transmission in the second, and the third-time slots depend on the cooperation scheme, DF or AF. The three-time slots DF scenario is investigated in Section "DF orthogonal three-time slots scenario", and the three-time slots AF scenario is investigated in Section "AF orthogonal three-time slots scenario". 


\section{DF orthogonal three-time slots scenario}

As illustrated in Table 1, the received signal $y_{T_{k j}}^{(\mathrm{DF})}$ at the destination node in time slot $T_{k}$ for DF scheme is as follows:

$$
y_{T_{k j}}^{(\mathrm{DF})}=\sqrt{\frac{P_{S}}{2}} h_{\mathrm{RD}}^{(i)} \tilde{x}_{i j}+n_{k j}^{(\mathrm{DF})}, \quad i=0,1, j=1,2 \& k=2,3,
$$

where $n_{k j}^{(\mathrm{DF})}$ is the received noise at the destination node in time slot $T_{k}$, and $\tilde{x}_{i j}$ is the $j$ th user decoded symbol at the $i$ th relay. The $i$ th ordered relay transmits with power $P_{R}^{(i)}$ equals half the source power $\left(P_{R}^{(i)}=P_{S} / 2\right)$ for each user. Defining $\gamma^{(0.5 D)}$ as the instantaneous SNR for the $j$ th user at the destination node after using MRC, and assuming the relays $R_{b 0}$ and $R_{b 1}$ decoded the symbol $x_{j}$ correctly (i.e., $\left.\tilde{x}_{0 j}=\tilde{x}_{1 j}=x_{j}\right)$, then $\gamma^{(0.5 \mathrm{DF})}$ is obtained as:

$$
\gamma^{(0.5 \mathrm{DF})}=\gamma_{S_{j} D}+\frac{1}{2} \gamma_{\mathrm{sum}}^{(\mathrm{DF})}
$$

where $\gamma_{\text {sum }}^{(\mathrm{DF})}$, is defined as $\gamma_{\text {sum }}^{(\mathrm{DF})}=\gamma_{b 0}^{(\mathrm{DF})}+\gamma_{b 1}^{(\mathrm{DF})}$, and $\gamma_{b 0}^{(\mathrm{DF})}, \gamma_{b 1}^{(\mathrm{DF})}$ are the instantaneous end-to-end SNR of the best and the next-best relays, respectively. The factor $\frac{1}{2}$ in (5) is due to the fact that the best and next-best relays are shared between the two users with equal power, where $P_{R}^{(i)}=P_{S} / 2$ for $i \in\{0,1\}$.

The best relay $R_{b 0}$ is the relay with the maximum instantaneous end-to-end SNR at the destination node, i.e., $\gamma_{b 0}^{(\mathrm{DF})}=\max _{l}\left(\gamma_{0}\left|h_{R_{l} D}\right|^{2}\right)$. The next-best relay $R_{b 1}$ is the relay with the next-maximum instantaneous endto-end SNR at the destination node, i.e., $\gamma_{b 1}^{(\mathrm{DF})}=$ $\max _{l, l \neq b 0}\left(\gamma_{0}\left|h_{R_{l} D}\right|^{2}\right)$, where $l=1, \ldots, N(l$ is used as an index for the relay without ordering). The selection of the best relay $R_{b 0}$ and next-best relay $R_{b 1}$ from the $N$ available relays is determined by ordering the instantaneous endto-end SNRs from the $N$ relays as follows $\gamma_{b 0}^{(\mathrm{DF})}>\gamma_{b 1}^{(\mathrm{DF})}>$ $\gamma_{b 2}^{(\mathrm{DF})}>\cdots>\gamma_{b N-1}^{(\mathrm{DF})}{ }^{\mathrm{a}}$ In the following, the probability density function (PDF) and the MGF of the end-to-end SNR $\gamma^{(0.5 D)}$ are derived in order to evaluate the BEP and outage probability performances of the proposed scenario.

In order to find the PDF $f_{\gamma_{\text {sum }}^{(\mathrm{DF})}}(z)$ of the instantaneous end-to-end SNR $\gamma_{\text {sum }}^{(\mathrm{DF})}$ from the best and the next-best relays, we consider the following. Instead of dealing with the decoding set $C$ as in [5], we assume that the relay is selected from the $N$ available relays. However, if a relay cannot decode the message correctly, it will not transmit and hence, the instantaneous end-to-end SNR will be set to zero [25]. The $l$ th relay can decode the message of the source $S_{j}$ if $\gamma_{0}\left|h_{S_{j} R_{l}}\right|^{2}$ is greater than some threshold value $\Delta_{\mathrm{TH}}$, i.e., if $\gamma_{0}\left|h_{S_{i} R_{l}}\right|^{2}>\Delta_{\mathrm{TH}}$. Defining $\beta$ as the probability of erroneously decoding the message, then $\beta$ is computed as $\beta=\operatorname{Pr}\left(\gamma_{0}\left|h_{S_{j} R_{l}}\right|^{2}<\Delta_{\mathrm{TH}}\right)=1-$ $e^{-\lambda_{\mathrm{SR}} \Delta_{\mathrm{TH}}}$, where $\gamma_{0}\left|h_{S_{j} R_{l}}\right|^{2}$ is an exponential random variable random variable with a parameter $\lambda_{\mathrm{SR}}$. Assuming the identical random variables, then $\Delta_{\mathrm{TH}}$ can be computed as the value of $\gamma_{0}\left|h_{S_{j} R_{l}}\right|^{2}$ that is sufficient to satisfy a given transmission rate $R$. In other words, $\Delta_{\mathrm{TH}}$ is the threshold value satisfying the inequality $\frac{1}{2} \log \left(1+\gamma_{0}\left|h_{S_{i} R_{l}}\right|^{2}\right) \geq R$ or equivalently $\gamma_{0}\left|h_{S_{j} R_{l}}\right|^{2} \geq \Delta_{\mathrm{TH}}=\left(2^{2 R}-1\right)$. The relay $R_{l}$ that satisfies this condition is considered in the decoding set $C[25,26]$. Defining the unordered instantaneous endto-end SNR at the destination node from the $l$ th relay as $\gamma_{l}^{(\mathrm{DF})}, \forall l=\{1, \ldots, N\}, \gamma_{l}^{(\mathrm{DF})}$ is then obtained as:

$$
\gamma_{l}^{(\mathrm{DF})}=\left\{\begin{array}{cl}
0, & \text { if } \gamma_{0}\left|h_{S_{j} R_{l}}\right|^{2}<\Delta_{\mathrm{TH}} . \\
\gamma_{0}\left|h_{R_{l} D}\right|^{2}, & \text { if } \gamma_{0}\left|h_{S_{j} R_{l}}\right|^{2} \geq \Delta_{\mathrm{TH}} .
\end{array}\right.
$$

The PDF $f_{\gamma_{l}^{(\mathrm{DF})}}(x)$, and the cumulative density function $(\mathrm{CDF}) F_{\gamma_{l}^{(\mathrm{DF})}}(x)$ of $\gamma_{l}^{(\mathrm{DF})}$ are given as [17]:

$$
\begin{aligned}
& f_{\gamma_{l}^{(\mathrm{DF})}}(x)=(1-\beta) \lambda_{\mathrm{RD}} e^{-\lambda_{\mathrm{RD}} x} \mu(x)+\beta \delta(x), \\
& F_{\gamma_{l}^{(\mathrm{DF})}}(x)=\left(1-(1-\beta) e^{-\lambda_{\mathrm{RD}} x}\right) \mu(x),
\end{aligned}
$$

where $\mu(x)$ is the unit step function, $\delta(x)$ is Dirac's delta function, and $\lambda_{\mathrm{RD}}$ is the parameter of the exponential random variable characterizing the received SNR at the destination node from the relay. All relay-destination links are assumed to be iid random variables with $\lambda_{\mathrm{RD}}=$ $\frac{1}{\gamma_{0} E\left\{\left|h_{R_{l} D}\right|^{2}\right\}}$.

In order to find the PDF of the SNR of the best and next-best relays, we refer to the order statistics of random variables. Let $X_{1}, X_{2}, \ldots, X_{N}$ be defined as iid random variables with PDF $f_{X}(x)$ and $\operatorname{CDF} F_{X}(x)$. In addition, define the ordered random variables $Y_{1}<Y_{2} \cdots<Y_{N}$, where $Y_{1}=\min _{l} X_{l}$, and $Y_{N}=\max _{l} X_{l}$ for $l \in\{1, \ldots N\}$ then the PDF of the $k$ th ordered random variable $Y_{k}$ is obtained as [27]:

$$
f_{Y_{k}}(y)=\left(\begin{array}{c}
N \\
k
\end{array}\right) k\left[F_{X}(y)\right]^{k-1}\left[1-F_{X}(y)\right]^{N-k} f_{X}(y),
$$

and the joint PDF of the two ordered random variables $\left(Y_{r}\right.$ and $Y_{s}$ ), where $r<s$ is given by [28]:

$$
\begin{aligned}
f_{Y_{r}, Y_{s}}(x, y)= & \frac{N !}{(r-1) !(s-r-1) !(N-s) !} \\
& \times\left[F_{X}(x)\right]^{r-1} f_{X}(x) f_{X}(y)\left[1-F_{X}(y)\right]^{N-s} \\
& \left.\times\left[F_{X}(y)-F_{X}(x)\right)\right]^{s-r-1} .
\end{aligned}
$$


From (8) the PDFs of the received SNR $\gamma_{b 0}^{(\mathrm{DF})}$, and $\gamma_{b 1}^{(\mathrm{DF})}$ from the best, and next-best relays are, respectively obtained as:

$$
\begin{aligned}
& f_{\gamma_{b 0}^{(\mathrm{DF})}}(z)=N\left[F_{X}(z)\right]^{N-1} f_{X}(z), \\
& f_{\gamma_{b 1}^{(\mathrm{DF})}}(z)=N(N-1)\left[F_{X}(z)\right]^{N-2}\left[1-F_{X}(z)\right] f_{X}(z) .
\end{aligned}
$$

The joint PDF of the received SNR from the ordered relays $\left(R_{b 0}, R_{b 1}\right)$ using $(9)$ is obtained as:

$$
f_{\gamma_{b 1}^{(\mathrm{DF})}, \gamma_{b 0}^{(\mathrm{DF})}}(x, y)=N(N-1)\left[F_{X}(x)\right]^{N-2} f_{X}(x) f_{X}(y) .
$$

Substituting (6) in (12), results in:

$$
\begin{aligned}
f_{\gamma_{b 1}^{(\mathrm{DF})}, \gamma_{b 0}^{(\mathrm{DF})}(x, y)}= & N(N-1)\left[\left(1-(1-\beta) e^{-\lambda_{\mathrm{RD}} x}\right) \mu(x)\right]^{N-2} \\
& \times\left((1-\beta) \lambda_{\mathrm{RD}} e^{-\lambda_{\mathrm{RD}} x} \mu(x)+\beta \delta(x)\right) \\
& \times\left((1-\beta) \lambda_{\mathrm{RD}} e^{-\lambda_{\mathrm{RD}} y} \mu(y)+\beta \delta(y)\right) .
\end{aligned}
$$

Note that, $\gamma_{b 0}^{(\mathrm{DF})}$, and $\gamma_{b 1}^{(\mathrm{DF})}$ are dependent random variables due to the ordering of the instantaneous end-to-end SNR. The PDF of their sum $\gamma_{\text {sum }}^{(\mathrm{DF})}=\gamma_{b 0}^{(\mathrm{DF})}+\gamma_{b 1}^{(\mathrm{DF})}$ is computed as [29]:

$$
\begin{aligned}
f_{\gamma_{\text {sum }}^{(\mathrm{DF})}}(z)= & A^{(\mathrm{DF})} e^{-\lambda_{\mathrm{RD}} z} \mu(z)+B^{(\mathrm{DF})} z e^{-\lambda_{\mathrm{RD}} z} \mu(z) \\
& +D^{(\mathrm{DF})} \delta(z)+\sum_{k=3}^{N} E_{k}^{(\mathrm{DF})} e^{-k \lambda_{\mathrm{RD}} \frac{z}{2}} \mu(z) .
\end{aligned}
$$

Defining $c_{k}$ as:

$$
c_{k}=\left(\begin{array}{c}
N \\
k
\end{array}\right) k(k-1)(-1)^{k-2}
$$

then $A^{(\mathrm{DF})}, B^{(\mathrm{DF})}, D^{(\mathrm{DF})}$, and $E^{(\mathrm{DF})}$ are computed as given in column 2 of Table 2 .
The MGF $\Psi_{\gamma_{\text {sum }}^{(\mathrm{DF})}}(s)$ of the received SNR $\gamma_{\text {sum }}^{(\mathrm{DF})}$ is obtained using (14) as:

$$
\begin{aligned}
\Psi_{\gamma_{\mathrm{sum}}^{(\mathrm{DF})}}(s)= & E\left\{e^{-s \gamma_{\mathrm{sum}}^{(\mathrm{DF})}}\right\}=\frac{A^{(\mathrm{DF})}}{s+\lambda_{\mathrm{RD}}}+\frac{B^{(\mathrm{DF})}}{\left(s+\lambda_{\mathrm{RD}}\right)^{2}} \\
& +\sum_{k=3}^{N} \frac{E_{k}^{(\mathrm{DF})}}{s+\frac{k \lambda_{\mathrm{RD}}}{2}}+D^{(\mathrm{DF})} .
\end{aligned}
$$

For the case of sharing the best and next-best relays, it is required to compute the MGF of $0.5 \gamma_{\text {sum }}^{(\mathrm{DF})}$, which can be obtained simply from $\gamma_{\text {sum }}^{(\mathrm{DF})}$ as $\Psi_{\frac{\gamma_{\text {sum }}}{2}}^{\text {(D) }}(s)=\Psi_{\gamma_{\text {sum }}^{(\mathrm{DF})}}\left(\frac{s}{2}\right)$. Since the received SNR from the source-destination link $\gamma_{S_{j} D}$ is independent of the received SNR from the relaydestination link $0.5 \gamma_{\text {sum }}^{(\mathrm{DF})}$, the MGF $\Psi_{\gamma^{(0.5 \mathrm{DF})}}(s)$ of the received SNR at the destination node after using MRC is $\Psi_{\gamma^{(0.5 \mathrm{DF})}}(s)=\Psi_{\gamma_{\text {sum }}^{(\mathrm{DF})}}\left(\frac{s}{2}\right) \Psi_{\gamma_{\mathrm{SD}}}(s)$. Applying the partial fraction expansion, we arrive at:

$$
\begin{aligned}
\Psi_{\gamma^{(0.5 \mathrm{DF})}}(s)= & \frac{a^{(\mathrm{DF})}}{1+\frac{s}{2 \lambda_{\mathrm{RD}}}}+\frac{b^{(\mathrm{DF})}}{\left(1+\frac{s}{2 \lambda_{\mathrm{RD}}}\right)^{2}} \\
& +\sum_{k=3}^{N} \frac{e_{k}^{(\mathrm{DF})}}{1+\frac{s}{\lambda_{\mathrm{RD}} k}}+\frac{f^{(\mathrm{DF})}}{1+\frac{s}{\lambda_{\mathrm{SD}}}},
\end{aligned}
$$

where $a^{(\mathrm{DF})}, b^{(\mathrm{DF})}, f^{(\mathrm{DF})}$, and $e_{k}^{(\mathrm{DF})}$ (assuming that $\lambda_{\mathrm{SD}} \neq$

\begin{tabular}{|c|c|c|c|}
\hline Coeff. & Value & Coeff. & Value \\
\hline$A^{(\mathrm{DF})}$ & $\begin{array}{c}\sum_{k=3}^{N} \lambda_{\mathrm{RD}} c_{k}(1-\beta)^{k-1}\left(\beta+\frac{1-\beta}{k-2}\right) \\
+c_{2}(1-\beta) \beta \lambda_{\mathrm{RD}}\end{array}$ & $a^{(\mathrm{DF})}$ & $\frac{1}{2 \lambda_{\mathrm{RD}}}\left(\frac{2 A^{(\mathrm{DF})} \lambda_{\mathrm{SD}}}{-2 \lambda_{\mathrm{RD}}+\lambda_{\mathrm{SD}}}-\frac{4 B^{(\mathrm{DF})} \lambda_{\mathrm{SD}}}{\left(-2 \lambda_{\mathrm{RD}}+\lambda_{\mathrm{SD}}\right)^{2}}\right.$ \\
\hline$B^{(\mathrm{DF})}$ & $\frac{c_{2}}{2}(1-\beta)^{2} \lambda_{\mathrm{RD}}^{2}$ & $b^{(\mathrm{DF})}$ & $\frac{1}{4 \lambda_{\mathrm{RD}}^{2}}\left(\frac{4 B^{(\mathrm{DF})} \lambda_{\mathrm{SD}}}{-2 \lambda_{\mathrm{RD}}+\lambda_{\mathrm{SD}}}\right)$ \\
\hline$D^{(\mathrm{DF})}$ & $\sum_{k=2}^{N} c_{k} \beta^{2}(1-\beta)^{k-2}$ & $f^{(\mathrm{DF})}$ & $\begin{array}{l}\frac{2 A^{(\mathrm{DF})}}{2 \lambda_{\mathrm{RD}}-\lambda_{\mathrm{SD}}}+\frac{4 B^{(\mathrm{DF})}}{\left(2 \lambda_{\mathrm{RD}}-\lambda_{\mathrm{SD}}\right)^{2}}+ \\
\sum_{k=3}^{N} \frac{2 E_{k}^{(\mathrm{DF})}}{\lambda_{\mathrm{RD}} k-\lambda_{\mathrm{SD}}}+D^{(\mathrm{DF})}\end{array}$ \\
\hline$E_{k}^{(\mathrm{DF})}$ & $\frac{c_{k}}{2}(1-\beta)^{k}\left(\frac{-2}{k-2}\right) \lambda_{\mathrm{RD}}$ & $e_{k}^{(\mathrm{DF})}$ & $\frac{1}{\lambda_{\mathrm{RD}} k}\left(\frac{2 E_{k}^{(\mathrm{DF})} \lambda_{\mathrm{SD}}}{-\lambda_{\mathrm{RD}} k+\lambda_{\mathrm{SD}}}\right)$ \\
\hline
\end{tabular}
$\lambda_{\mathrm{RD}}$ or multiple of it for simplicity, but the analysis can be easily extended), are given as in column 4 of Table 2 .

Since MRC is used at the destination node, the SEP can be calculated by averaging the multichannel conditional SEP over the PDF of the random variable representing the received end-to-end SNR at the destination node [14]. The SEP for MPSK and MQAM are respectively obtained by using the MGF $\Psi(s)$ of the received end-to-end SNR as follows [30]:

$$
\mathrm{SEP}_{\text {MPSK }}=\frac{1}{\pi} \int_{0}^{\frac{(M-1) \pi}{M}} \Psi\left(\frac{\sin ^{2}\left(\frac{\pi}{M}\right)}{\sin ^{2} \theta}\right) d \theta
$$

Table 2 Coefficients of the PDF, the MGF, the BEP, and the $P_{\text {out }}$ for DF sharing scheme 


$$
\begin{aligned}
\operatorname{SEP}_{M Q A M}= & \frac{4 q}{\pi} \int_{0}^{\frac{\pi}{2}} \Psi\left(\frac{3}{2(M-1) \sin ^{2} \theta}\right) d \theta \\
& -\frac{4 q^{2}}{\pi} \int_{0}^{\frac{\pi}{4}} \Psi\left(\frac{3}{2(M-1) \sin ^{2} \theta}\right) d \theta
\end{aligned}
$$

where $q=1-\frac{1}{\sqrt{M}}$. In this article, the SEP is calculated only for BPSK modulation by substituting $M=2$ in (18), but it can be easily extended to MPSK, and MQAM using (18) and (19), respectively, and can be expressed in a closed-form using the hypergeometric function [30]. In the following we obtain the BEP for different sharing scenarios. The BEP is computed using (18) as:

$$
\mathrm{BEP}=\frac{1}{\pi} \int_{0}^{\frac{\pi}{2}} \Psi\left(\frac{1}{\sin ^{2} \theta}\right) d \theta
$$

Defining the function $F^{n}(u)$ as:

$$
\begin{aligned}
F^{n}(u) & =\frac{1}{\pi} \int_{0}^{\frac{\pi}{2}} \frac{1}{\left(1+\frac{u}{\sin ^{2} \theta}\right)^{n}} d \theta \\
& =\left(\frac{1-k_{u}}{2}\right)^{n} \sum_{t=0}^{n-1}\left(\begin{array}{c}
n-1+t \\
t
\end{array}\right)\left(\frac{1+k_{u}}{2}\right)^{t},
\end{aligned}
$$

where $k_{u}=\sqrt{\frac{u}{1+u}}$ and $n$ is an integer. The integral for $n=1$ simplifies to $F^{1}(u)=\frac{1}{2}\left(1-k_{u}\right)$. The $\mathrm{BEP}_{\gamma^{(0.5 \mathrm{DF})}}$ is obtained by substituting (17) into (20), which can be computed as follows:

$$
\begin{aligned}
\mathrm{BEP}_{\gamma^{(0.5 \mathrm{DF})}=} & \frac{a^{(\mathrm{DF})}}{2}\left(1-\sqrt{\frac{1}{1+2 \lambda_{\mathrm{RD}}}}\right)+b^{(\mathrm{DF})} F^{2}\left(\frac{1}{2 \lambda_{\mathrm{RD}}}\right) \\
& +\sum_{k=3}^{N} \frac{e_{k}^{(\mathrm{DF})}}{2}\left(1-\sqrt{\frac{1}{1+\lambda_{\mathrm{RD}} k}}\right) \\
& +\frac{f^{(\mathrm{DF})}}{2}\left(1-\sqrt{\frac{1}{1+\lambda_{\mathrm{SD}}}}\right) .
\end{aligned}
$$

The outage probability $P_{\text {out }}$ is defined as the probability of the end-to-end SNR $\Gamma$ when it falls below a threshold value $\gamma_{\mathrm{TH}}$, and computed as:

$$
P_{\text {out }}=\int_{0}^{\gamma_{\mathrm{TH}}} f_{\Gamma}(\gamma) d \gamma=F_{\Gamma}\left(\gamma_{\mathrm{TH}}\right)
$$

For the two best ordered relays DF sharing scenario, the CDF $F_{\gamma^{(0.5 D F)}}(x)$ of the end-to-end SNR $\gamma^{(0.5 \mathrm{DF})}$ can be easily obtained from the MGF $\Psi_{\gamma^{(0.5 D F)}}(s)$ given in (17).
The outage probability $P_{\text {out }}^{(0.5 \mathrm{DF})}$ can then be formulated as:

$$
\begin{aligned}
P_{\mathrm{out}}^{(0.5 \mathrm{DF})}=F_{\gamma^{(0.5 \mathrm{DF})}\left(\gamma_{\mathrm{TH}}\right)=} & \sum_{k=3}^{N} e_{k}^{(\mathrm{DF})}\left(1-e^{-\lambda_{\mathrm{RD}} k \gamma_{\mathrm{TH}}}\right) \\
& +f^{(\mathrm{DF})}\left(1-e^{-\lambda_{\mathrm{SD}} \gamma_{\mathrm{TH}}}\right) \\
& +\left(a^{(\mathrm{DF})}+b^{(\mathrm{DF})}\right)\left(1-e^{-2 \lambda_{\mathrm{RD}} \gamma_{\mathrm{TH}}}\right) \\
& -2 \lambda_{\mathrm{RD}} b^{(\mathrm{DF})} \gamma_{\mathrm{TH}}\left(e^{-2 \lambda_{\mathrm{RD}} \gamma_{\mathrm{TH}}}\right) .
\end{aligned}
$$

The diversity order of sharing the two ordered best relays can be investigated using asymptotic analysis of the BEP or the outage probability $P_{\text {out }}$ at high SNR values $[3,13]$. Another approach, is to use the asymptotic analysis of the PDF or the MGF of the end-to-end SNR [31-33]. We follow the latter approach using the MGF of the endto-end SNR at the output of the MRC. Using the results of [31], the MGF can be approximated as $s \rightarrow \infty$ by $b|s|^{-d}+O\left(|s|^{-d}\right)$, $^{\mathrm{b}}$ where $d$ is the diversity order, and $b$

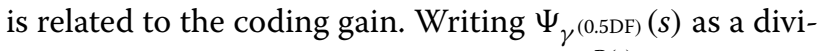
sion of two polynomials $\Psi_{\gamma^{(0.5 \mathrm{DF})}(s)}=\frac{B(s)}{A(s)}$, where $B(s)$ and $A(s)$ are the numerator and denominator polynomials, respectively. $A(s)$ can be written as, $A(s)=(1+$ $\left.\frac{s}{2 \lambda_{\mathrm{RD}}}\right)^{2}\left(1+\frac{s}{\lambda_{\mathrm{SD}}}\right) \prod_{k=3}^{N}\left(1+\frac{s}{\lambda_{\mathrm{RD}}}\right)$, which can be approximated for $s \rightarrow \infty$ as $A(s) \approx\left(\frac{s}{2 \lambda_{\mathrm{RD}}}\right)^{2}\left(\frac{s}{\lambda_{\mathrm{SD}}}\right) \prod_{k=3}^{N}\left(\frac{s}{\lambda_{\mathrm{RD}} k}\right)=$ $\left(2 \lambda_{\mathrm{RD}} \lambda_{\mathrm{SD}} \prod_{k=3}^{N} \lambda_{\mathrm{RD}} k\right)^{-1} s^{(N+1)}$. The numerator polynomial can be found by collecting and combining the corresponding terms, which is clearly of degree less than the denominator polynomial. Taking only the constant term of the numerator polynomial, and divide this term by the approximation of the denominator polynomial results in the term $b|s|^{-(N+1)}$. This means that the diversity order is $N+1$. Other terms which result from the division of the numerator polynomial with the approximation of the denominator polynomial contribute to $O\left(|s|^{-(N+1)}\right)$.

\section{AF orthogonal three-time slots scenario}

As illustrated in Table 1 , the received signal $y_{T_{k j}}^{(\mathrm{AF})}$ at the destination node in time slot $T_{k}$ for AF scheme is as follows:

$$
\begin{aligned}
y_{T_{k j}}^{(\mathrm{AF})}= & G_{S_{j} R}^{(i)} \sqrt{\frac{P_{S}}{2}} h_{\mathrm{RD}}^{(i)} y_{T_{1 j}}^{\left(R_{b i}\right)} \\
& +n_{k j}^{(\mathrm{AF})}, \quad i=0,1, j=1,2 \& k=2,3,
\end{aligned}
$$

where $n_{k j}^{(\mathrm{AF})}$ is the additive noise at the destination, and $G_{S_{j} R}^{(i)}$ is the normalizing factor at the relay, which depends on the instantaneous CSI between the $j$ th source and the 
$i$ th ordered best relay. Assuming that each relay knows its instantaneous channel information $h_{S_{j} R}^{(i)}$, the normalizing factor using (3) is $G_{S_{j} R}^{(i)}=\frac{1}{\sqrt{P_{S}\left|h_{S_{j} R}^{(i)}\right|^{2}+N_{0}}}$

The end-to-end received SNR at the destination node for AF scheme (with instantaneous CSI at the $l$ th relay) with $P_{R_{l}}=P_{S} / 2$, and using the normalizing factor $G_{S_{j} R_{l}}=$ $\frac{1}{\sqrt{P_{S}\left|h_{S_{j} R_{l}}\right|^{2}+N_{0}}}$ is obtained as:

$$
\gamma_{l}^{(\mathrm{AF})}=\frac{\frac{\gamma_{S_{j} R_{l}} \gamma_{R_{l} D}}{2}}{1+\gamma_{S j R_{l}}+\frac{\gamma_{R_{l} D}}{2}},
$$

which can be upper and lower bounded as [7,34]:

$$
\frac{1}{2} \min \left(\gamma_{S_{j} R_{l}}, \frac{\gamma_{R_{l} D}}{2}\right) \leq \gamma_{l}^{(\mathrm{AF})} \leq \min \left(\gamma_{S_{j} R_{l}}, \frac{\gamma_{R_{l} D}}{2}\right) .
$$

The upper bound in (27) is shown to be tight, and can be used to simplify the analysis [22]. It is easy to show that the PDF of the upper bound in (27) for Raleigh flat fading channels is an exponential random variable with parameter $\lambda_{\mathrm{eq}}=\lambda_{\mathrm{SR}}+2 \lambda_{\mathrm{RD}}$. The upper bound of the received end-to-end SNR $\gamma^{(0.5 \mathrm{AF})}$ at the destination node after using MRC, and using (25) and (3) is given as:

$$
\gamma^{(0.5 \mathrm{AF})}=\gamma_{S_{j} D}+\gamma_{\mathrm{sum}}^{(0.5 \mathrm{AF})}
$$

where $\gamma_{\text {sum }}^{(0.5 \mathrm{AF})}=\gamma_{b 0}^{(0.5 \mathrm{AF})}+\gamma_{b 1}^{(0.5 \mathrm{AF})}$ with $\gamma_{b 0}^{(0.5 \mathrm{AF})}$ and $\gamma_{b 1}^{(0.5 \mathrm{AF})}$ are the upper bound of the end-to-end SNR from the best, and the next-best relays respectively. The best relay $R_{b 0}$ is selected as the relay with the maximum upper bound of the end-to-end SNR $\gamma_{b 0}^{(0.5 \mathrm{AF})}$ at the the destination node, i.e., $\gamma_{b 0}^{(0.5 \mathrm{AF})}=\max _{l}\left(\min \left(\gamma_{S_{j} R_{l}}, \frac{\gamma_{R_{l} D}}{2}\right)\right)$, where $l=1, \ldots, N$. Similarly, the next-best relay $R_{b 1}$ is selected as the relay with the next-maximum upper bound of the end-to-end SNR $\gamma_{b 1}^{(0.5 \mathrm{AF})}$ at the the destination node, i.e., $\gamma_{b 1}^{(0.5 \mathrm{AF})}=\max _{l, l \neq b 0}\left(\min \left(\gamma_{S_{j} R_{l}}, \frac{\gamma_{R_{l} D}}{2}\right)\right)$. The selection of the best and next-best relays $R_{b 0}$ and $R_{b 1}$, respectively from the $N$ available relays is done using the ordering of the upper bound of end-to-end SNRs from the $N$ available relays as: $\gamma_{b 0}^{(0.5 \mathrm{AF})}>\gamma_{b 1}^{(0.5 \mathrm{AF})}>\gamma_{b 2}^{(0.5 \mathrm{AF})}>\cdots>\gamma_{b N-1}^{(0.5 \mathrm{AF})}$.
The joint PDF of the upper bound received SNRs from the ordered relays $R_{b 0}$ and $R_{b 1}$ using (9) can then be obtained as:

$$
\begin{aligned}
f_{\gamma_{b 1}^{(0.5 \mathrm{AF})}, \gamma_{b 0}^{(0.5 \mathrm{AF})}(x, y)=} & N(N-1)\left[\left(1-e^{\left.-\lambda_{\text {eq }} x^{x}\right)}\right]^{N-2}\right. \\
& \times \lambda_{\text {eq }}^{2} e^{-\lambda_{\text {eq }}{ }^{x}} e^{-\lambda_{\text {eq }} y} \mu(x) \mu(y) .
\end{aligned}
$$

Using (29), and following a similar procedure to that followed in Section "DF orthogonal three-time slots scenario", the $\mathrm{BEP}_{\gamma(0.5 \mathrm{AF})}$ can be derived as:

$$
\begin{aligned}
\mathrm{BEP}_{\gamma(0.5 \mathrm{AF})=} & \frac{a^{(\mathrm{AF})}}{2}\left(1-\sqrt{\frac{1}{1+\lambda_{\mathrm{eq}}}}\right)+b^{(\mathrm{AF})} F^{2}\left(\frac{1}{\lambda_{\mathrm{eq}}}\right) \\
& +\sum_{k=3}^{N} \frac{e_{k}^{(\mathrm{AF})}}{2}\left(1-\sqrt{\frac{1}{1+\frac{\lambda_{\mathrm{eq}} k}{2}}}\right) \\
& +\frac{f^{(\mathrm{AF})}}{2}\left(1-\sqrt{\frac{1}{1+\lambda_{\mathrm{SD}}}}\right)
\end{aligned}
$$

where $A^{(\mathrm{AF})}, B^{(\mathrm{AF})}$, and $E_{k}^{(\mathrm{AF})}$ are defined in column 2 of Table 3 , and $a^{(\mathrm{AF})}, b^{(\mathrm{AF})}, e_{k}^{(\mathrm{AF})}$ and $f^{(\mathrm{AF})}$ are defined in column 4 of Table 3 . The outage probability $P_{\text {out }}^{(0.5 \mathrm{AF})}$ of sharing the two ordered best relays for AF scheme is then found using (23) as:

$$
\begin{aligned}
P_{\mathrm{out}}^{(0.5 \mathrm{AF})}=F_{\gamma^{(0.5 \mathrm{AF})}}\left(\gamma_{\mathrm{TH}}\right) & =\sum_{k=3}^{N} e_{k}^{(\mathrm{AF})}\left(1-e^{-\frac{\lambda_{\mathrm{eq}} k}{2} \gamma_{\mathrm{TH}}}\right) \\
& +f^{(\mathrm{AF})}\left(1-e^{-\lambda_{\mathrm{SD}} \gamma_{\mathrm{TH}}}\right) \\
& +\left(a^{(\mathrm{AF})}+b^{(\mathrm{AF})}\right)\left(1-e^{-\lambda_{\mathrm{eq}} \gamma_{\mathrm{TH}}}\right) \\
& -\lambda_{\text {eq }} b^{(\mathrm{AF})} \gamma_{\mathrm{TH}}\left(e^{-\lambda_{\mathrm{eq}} \gamma_{\mathrm{TH}}}\right)
\end{aligned}
$$

The diversity order of sharing the two ordered best

\begin{tabular}{|c|c|c|c|}
\hline Coeff. & Value & Coeff. & Value \\
\hline$A^{(\mathrm{AF})}$ & $\sum_{k=3}^{N} c_{k} \lambda_{\text {eq }} \frac{1}{k-2}$ & $a^{(\mathrm{AF})}$ & $\frac{1}{\lambda_{\text {eq }}}\left(\frac{A^{(\mathrm{AF})} \lambda_{\mathrm{SD}}}{-\lambda_{\text {eq }}+\lambda_{\mathrm{SD}}}-\frac{B^{(\mathrm{AF})} \lambda_{\mathrm{SD}}}{\left(-\lambda_{\text {eq }}+\lambda_{\mathrm{SD}}\right)^{2}}\right)$ \\
\hline$B^{(\mathrm{AF})}$ & $\frac{c_{2} \lambda_{\text {eq }}^{2}}{2}$ & $b^{(\mathrm{AF})}$ & $\frac{1}{\lambda_{\mathrm{eq}}^{2}}\left(\frac{B^{(\mathrm{AF})} \lambda_{\mathrm{SD}}}{-\lambda_{\mathrm{eq}}+\lambda_{\mathrm{SD}}}\right)$ \\
\hline \multirow[t]{2}{*}{$E_{k}^{(\mathrm{AF})}$} & $-\frac{c_{k} \lambda_{\mathrm{eq}}}{k-2}$ & $e_{k}^{(\mathrm{AF})}$ & $\frac{2}{\lambda_{\text {eq }} k}\left(\frac{E_{k}^{(A F)} \lambda_{S D}}{-\frac{\lambda \text { eqk }}{2}+\lambda_{\text {SD }}}\right)$ \\
\hline & & $f^{(\mathrm{AF})}$ & $\frac{A^{(A F)}}{\lambda_{\text {eq }}-\lambda_{S D}}+\frac{B^{(A F)}}{\left(\lambda_{\text {eq }}-\lambda_{S D}\right)^{2}}+\sum_{k=3}^{N} \frac{E_{k}^{(A F)}}{\left(\frac{\lambda_{e q} k}{2}-\lambda_{S D}\right)}$ \\
\hline
\end{tabular}
relays for $\mathrm{AF}$ scheme is also $N+1$, which can be found from the similarity between $\mathrm{BEP}_{\gamma}(0.5 \mathrm{AF})$ for $\mathrm{AF}$ sharing (30) and the $\mathrm{BEP}_{\gamma(0.5 \mathrm{DF})}$ for DF sharing (22).

It is worth noting that, for AF sharing scenario, the ordering of the best, and next-best relays depends on the relay's transmitted power with the assumption that the relays transmit with the same power level. Therefore, the best and next-best relays in this scenario are different from the best and next-best relays without sharing. In the sharing scenario, the best relay is selected as

Table 3 Coefficients of the PDF, the MGF, the BEP, and the $P_{\text {out }}$ for AF sharing scheme 
$\max _{l}\left(\min \left(\gamma_{S_{j} R_{l}}, \frac{\gamma_{R_{l} D}}{2}\right)\right)$ but the best relay without sharing is selected as $\max _{l}\left(\min \left(\gamma_{S_{j} R_{l}}, \gamma_{R_{l} D}\right)\right)$. It is clear that the factor $\frac{1}{2}$ affects the ordering of the relays. The same result holds for the next-best relay. In general the ordered best relays for sharing and without sharing AF are different (even the relays transmit with equal power). Whereas for DF scenario the relays are ordered depending on the received SNR at the destination as given by $\max _{l} \gamma_{0}\left|h_{\mathrm{RD}}^{(l)}\right|^{2}$ if the relays transmit with full power, and as $\max _{l} \frac{1}{2} \gamma_{0}\left|h_{\mathrm{RD}}^{(l)}\right|^{2}$ if the relays transmit with half power. The factor $\frac{1}{2}$ in the last expression does not affect the ordering of the relays, which can be removed from the expression without affecting the ordering. The different cases for AF ordering are illustrated in Table 4, the rows 3 and 4 illustrate that the sharing scenario may use different relays from the best and next-best relays which were ordered based on full power transmission. Simulation results show that AF sharing scenario based on half power allocation achieves full diversity order and outperforms the BEP performance of the best relay (alone) scenario. The ordering based on relay half power allocation is used for two reasons: First, the BEP performance of the sharing based on half power ordering outperforms the sharing based on full power ordering. Second, using half power ordering simplifies deriving the PDF expression (29).

\section{Scenario two (two-time slots scenario)}

So far, the transmission schemes discussed do not utilize the resources efficiently. The sources and the relays need to wait for three-time slots to start new transmission. In this section we discuss more efficient transmission schemes where only two time slots are required. Three types of such transmission schemes are discussed next. These schemes are DF with distributed STBC, DF, and AF with distributed BF.

\section{DF with distributed STBC (STBC-DF)}

In this scenario, the selected relays are shared between the two sources (users) equally. The two sources are assumed to have the same best and next-best relays. As illustrated in Table 5, only two-time slots are used for cooperation. Alamouti STBC is used between the relays and the destination node $[35,36]$. Denote $R_{T_{21}}^{(\mathrm{DS})}, R_{T_{22}}^{(\mathrm{DS})}$ as the noiseless

Table 4 Best relay selection criterion for AF scheme

\begin{tabular}{cccc}
\hline The case & $\begin{array}{c}\text { The best relay } \\
\text { with sharing }\end{array}$ & $\begin{array}{c}\text { The best relay } \\
\text { without sharing }\end{array}$ & Comments \\
\hline$\gamma_{S_{j} R_{l}}<\frac{\gamma_{R_{l} D}}{2}<\gamma_{R_{l} D}$ & $\max _{l}\left(\gamma_{S_{j} R_{l}}\right)$ & $\max _{1}\left(\gamma_{S_{j} R_{l}}\right)$ & Same \\
$\frac{\gamma_{R_{l} D}}{2}<\gamma_{S_{j} R_{l}}<\gamma_{R_{l} D}$ & $\max _{l}\left(\frac{\gamma_{R_{l} D}}{2}\right)$ & $\max _{l}\left(\gamma_{S_{j} R_{l}}\right)$ & Different \\
$\frac{\gamma_{R_{l} D}}{2}<\gamma_{R_{l} D}<\gamma_{S_{j} R_{l}}$ & $\max _{l}\left(\frac{\gamma_{R_{l} D}}{2}\right)$ & $\max _{1}\left(\gamma_{R_{l} D}\right)$ & Different \\
\hline
\end{tabular}

\section{Table 5 Distributed STBC-DF scenario}

\begin{tabular}{ccccc}
\hline & $T_{11}$ & $T_{\mathbf{1 2}}$ & $T_{\mathbf{2 1}}$ & $T_{\mathbf{2 2}}$ \\
\cline { 2 - 5 }$S_{1}$ & $T_{x_{1}}$ & - & - & - \\
$S_{2}$ & - & $T_{x_{2}}$ & - & - \\
$R_{b 0}$ & $R_{x_{1}}$ & $R_{x_{2}}$ & $T_{\tilde{x}_{1}}$ & $T_{-\tilde{x}_{2}^{*}}$ \\
$R_{b 1}$ & $R_{x_{1}}$ & $R_{x_{2}}$ & $T_{\tilde{x}_{2}}$ & $T_{\tilde{x}_{1}^{*}}$ \\
$D$ & $R_{x_{1}}$ & $R_{x_{2}}$ & $R_{T_{22}}^{(D S)}$ & $R_{T_{23}}^{(D S)}$ \\
\hline
\end{tabular}

received signals generated using distributed STBC in time sub-slots $T_{21}$ and $T_{22}$, respectively. The received signals $y_{21}^{(\mathrm{DS})}, y_{22}^{(\mathrm{DS})}$ at the destination node in time sub-slots $T_{21}$ and $T_{22}$, respectively, are given as:

$$
\begin{aligned}
& y_{21}^{(\mathrm{DS})}=\sqrt{\frac{P_{S}}{2}} h_{\mathrm{RD}}^{(0)} \tilde{x}_{01}+\sqrt{\frac{P_{S}}{2}} h_{\mathrm{RD}}^{(1)} \tilde{x}_{12}+n_{21}^{(\mathrm{DS})}, \\
& y_{22}^{(\mathrm{DS})}=-\sqrt{\frac{P_{S}}{2}} h_{\mathrm{RD}}^{(0)} \tilde{x}_{02}^{*}+\sqrt{\frac{P_{S}}{2}} h_{\mathrm{RD}}^{(1)} \tilde{x}_{11}^{*}+n_{22}^{(\mathrm{DS})},
\end{aligned}
$$

where $\tilde{x}_{i j}$ is the decoded symbol of the transmitted symbol $x_{j}$ at the best relay $R_{b i}$. The superscript ${ }^{*}$ stands for complex conjugate. $n_{2 j}^{(\mathrm{DS})}$ is the additive noise at the destination in time sub-slot $T_{2 j}$ for $j=1,2$. The estimated symbols $\hat{x}_{1}$ and $\hat{x}_{2}$ at the destination in time sub-slots $T_{21}$ and $T_{22}$ can be found with the assumption that the transmitted symbols are correctly decoded at the relays (i.e., $\left.\tilde{x}_{0 j}=\tilde{x}_{1 j}=x_{j}\right)$ as [35]:

$$
\begin{aligned}
& \hat{x}_{1}=h_{\mathrm{RD}}^{*(0)} y_{21}^{(\mathrm{DS})}+h_{\mathrm{RD}}^{(1)} y_{22}^{*(\mathrm{DS})}, \\
& \hat{x}_{2}=h_{\mathrm{RD}}^{*(1)} y_{21}^{(\mathrm{DS})}-h_{\mathrm{RD}}^{(0)} y_{22}^{*(\mathrm{DS})}
\end{aligned}
$$

Defining $\gamma_{\text {sum }}^{(\mathrm{DS})}=\gamma_{b 0}^{(\mathrm{DS})}+\gamma_{b 1}^{(\mathrm{DS})}$, the end-to-end SNR at the destination node after using MRC is obtained as $\gamma^{(\mathrm{DS})}=\gamma_{S_{j} D}+\gamma_{\text {sum }}^{(\mathrm{DS})}$, which is similar to the SNR expression obtained in (5). Hence, the same analysis can be carried out; the BEP performance is the same as (22). The goal of this analysis is not to investigate distributed STBC, but to examine the BEP performance of sharing the two ordered best relays. Detailed analysis of distributed STBC for multi-relay systems using pairwise error probability can be found in [37].

The two best order relays STBC-AF requires more investigation for the selection criterion, and the amplification gain at the relays using the instantaneous CSI. This however, is outside the scope of this article and will be a subject for further investigation.

\section{Distributed BF for DF scheme}

In this scenario, two-time slots are used as in the previous scenario, except that the best and the next-best relays transmit the same information at the same time, as illustrated in Table 6. Denote $R_{T_{21}}^{(\mathrm{BD})}$ and $R_{T_{22}}^{(\mathrm{BD})}$ as the noiseless 
Table 6 Distributed BF scenarios

\begin{tabular}{ccccc}
\hline & $T_{11}$ & $T_{12}$ & $T_{21}$ & $T_{22}$ \\
\cline { 2 - 5 }$S_{1}$ & $T_{x_{1}}$ & - & - & - \\
$S_{2}$ & - & $T_{x_{2}}$ & - & - \\
$R_{b 0}$ & $R_{x_{1}}$ & $R_{x_{2}}$ & $T_{\tilde{x}_{1}} / T_{x_{1}}$ & $T_{\tilde{x}_{2}} / T_{x_{2}}$ \\
$R_{b 1}$ & $R_{x_{1}}$ & $R_{x_{2}}$ & $T_{\tilde{x}_{1}} / T_{x_{1}}$ & $T_{\tilde{x}_{2}} / T_{x_{2}}$ \\
$D$ & $R_{x_{1}}$ & $R_{x_{2}}$ & $R_{T_{21}}^{(\mathrm{BD})} / R_{T_{21}}^{(\mathrm{BA})}$ & $R_{T_{22}}^{(\mathrm{BD})} / R_{T_{22}}^{(\mathrm{BA})}$ \\
\hline
\end{tabular}

received signals using DF distributed $\mathrm{BF}$ in time sub-slots $T_{21}, T_{22}$ respectively, the received signal at the destination node in the second-time slot for BF-DF (BD) is:

$$
y_{2 j}^{(\mathrm{BD})}=\sqrt{\frac{P_{S}}{2}}\left(\left|h_{\mathrm{RD}}^{(0)}\right| \tilde{x}_{j 0}+\left|h_{\mathrm{RD}}^{(1)}\right| \tilde{x}_{j 1}\right)+n_{2 j}^{(\mathrm{BD})} j=1,2,
$$

where $\tilde{x}_{j 0}$ and $\tilde{x}_{j 1}$ are the decoded symbols of the $j$ th user at the relays $R_{b 0}$ and $R_{b 1}$ respectively, and $n_{2 j}^{(\mathrm{BD})}$ is the received noise at the destination at time slot $T_{2}$. From (36) and using MRC with the assumption that the signal is decoded correctly at both relays $R_{b 0}$ and $R_{b 1}\left(\tilde{x}_{j 0}=\tilde{x}_{j 1}\right)$ the $\operatorname{SNR} \gamma^{(\mathrm{BD})}$ at the destination is given as:

$$
\gamma^{(\mathrm{BD})}=\gamma_{S_{j} D}+0.5\left(\sqrt{\left|\gamma_{b 0}^{(D B)}\right|}+\sqrt{\left|\gamma_{b 1}^{(D B)}\right|}\right)^{2}
$$

It is rather complicated to obtain the PDF and/or the MGF for $\gamma^{(\mathrm{BD})}$ in (37) at the destination node analytically. In this respect, we obtain the PDF and MGF of the bounded SNR as follows. Defining $\gamma_{B}=$ $0.5\left(\sqrt{\gamma_{b 0}^{(\mathrm{BD})}}+\sqrt{\gamma_{b 1}^{(\mathrm{BD})}}\right)^{2}$, then $\gamma_{B}$ can be lower and upper bounded as:

$$
\frac{1}{2}\left(\gamma_{b 0}^{(\mathrm{BD})}+3 \gamma_{b 1}^{(\mathrm{BD})}\right) \leq \gamma_{B} \leq \frac{1}{2}\left(3 \gamma_{b 0}^{(\mathrm{BD})}+\gamma_{b 1}^{(\mathrm{BD})}\right)
$$

Now, defining $Z_{1}=\frac{1}{2}\left(\gamma_{b 0}^{(\mathrm{BD})}+3 \gamma_{b 1}^{(\mathrm{BD})}\right)$ and $Z_{2}=$ $\frac{1}{2}\left(3 \gamma_{b 0}^{(\mathrm{BD})}+\gamma_{b 1}^{(\mathrm{BD})}\right)$, the PDF of $Z_{1}$, and the PDF of $Z_{2}$ can be easily computed using the joint PDF of $f_{\gamma_{b 1}^{(\mathrm{BD})}, \gamma_{b 0}^{(\mathrm{BD})}}(x, y)$ given in (13). The MGF of the lower and upper bounds follow easily. Based on the MGF $\Psi_{\gamma_{\mathrm{SD}}}(s) \Psi_{Z_{1}}(s)$ and the MGF $\Psi_{\gamma_{S_{j} D}}(s) \Psi_{Z_{2}}(s)$ (note that the random variables $\gamma_{S_{\mathrm{j}} D}$ and $Z_{1}$, and $\gamma_{S_{j} D}$ and $Z_{2}$ are independent) the upper and the lower bounds of the $\mathrm{BEP}^{(\mathrm{BD})}$ can be computed using (20).
Applying partial fraction expansion, the $\mathrm{BEP}^{(\mathrm{BD})}$ is upper bounded as:

$$
\begin{aligned}
\mathrm{BEP}^{(\mathrm{BD})} \leq & \frac{a^{(U)}}{2}\left(1-\sqrt{\frac{1}{1+\frac{2 \lambda_{\mathrm{RD}}}{3}}}\right)+\frac{f^{(U)}}{2}\left(1-\sqrt{\frac{1}{1+\lambda_{\mathrm{SD}}}}\right) \\
& +\sum_{k=2}^{N} \frac{e_{k}^{(U)}}{2}\left(1-\sqrt{\frac{1}{1+\frac{\lambda_{\mathrm{RD}} k}{2}}}\right)
\end{aligned}
$$

where $A^{(U)}, E_{k}^{(U)}, a^{(U)}, f^{(U)}$ and $e_{k}^{(U)}$ are as defined in column 2 of Table 7 . Similarly, the BEP ${ }^{(B D)}$ is lower bounded as:

$$
\begin{aligned}
\mathrm{BEP}^{(\mathrm{BD})} \geq & \frac{a^{(L)}}{2}\left(1-\sqrt{\frac{1}{1+2 \lambda_{\mathrm{RD}}}}\right)+b^{(L)} F^{2}\left(\frac{1}{2 \lambda_{\mathrm{RD}}}\right) \\
& +\frac{f^{(L)}}{2}\left(1-\sqrt{\frac{1}{1+\lambda \mathrm{SD}}}\right) \\
& +\sum_{\substack{k=2 \\
k \neq 4}}^{N} \frac{e_{k}^{(L)}}{2}\left(1-\sqrt{\frac{1}{1+\frac{\lambda_{\mathrm{RD}} k}{2}}}\right)
\end{aligned}
$$

where $A^{(L)}, B^{(L)}, E_{k}^{(L)}, a^{(L)}, f^{(L)}$, and $e_{k}^{(L)}$ are as defined in column 3 in Table 7.

A tighter upper bound for the end-to-end SNR $\gamma^{(\mathrm{BD})}$ can be obtained by comparison with the optimal power allocation under total power sum constraint as [38]:

$$
\gamma^{(\mathrm{BD})} \leq \gamma_{O p}^{(\mathrm{BD})}=\gamma_{S_{j} D}+\gamma_{b_{0}}^{(\mathrm{BD})}+\gamma_{b_{1}}^{(\mathrm{BD})}
$$

where $\gamma_{O p}^{(\mathrm{BD})}$ is defined as the maximum received SNR using optimum power assignment for distributed BF scenario using the best and the next-best relays under the constraint $P_{R}^{(0)}+P_{R}^{(1)}=P_{S}$. This upper bound can also be used to compute the $\mathrm{BEP}_{O p}^{(\mathrm{BD})}$ using (22) by replacing $2 \lambda_{\mathrm{RD}}$ with $\lambda_{\mathrm{RD}}$ in all terms. Hence, the $\mathrm{BEP}^{(\mathrm{BD})}$ can be lower bounded by $\mathrm{BEP}_{O p}^{(\mathrm{BD})}$, i.e., $\mathrm{BEP}_{O p}^{(\mathrm{BD})} \leq \mathrm{BEP}^{(\mathrm{BD})}$. However, in this article we are only concerned with equal power sharing to simplify the analysis.

\section{Distributed BF for AF scheme}

In this scenario, two-time slots are used as in the previous scenarios, as illustrated in Table 6. Denote $R_{T_{21}}^{(\mathrm{BA})}$ and $R_{T_{22}}^{(\mathrm{BA})}$ as the noiseless received signals using AF distributed BF in time sub-slots $T_{21}$ and $T_{22}$ respectively, the received signal 
Table 7 Coefficients of the PDF, the MGF and the BEP for the upper and the lower bounds of BD

\begin{tabular}{|c|c|c|}
\hline Coeff. & $S=U$ & $S=L$ \\
\hline$A^{(S)}$ & $\frac{\lambda_{\mathrm{RD}}}{3} \sum_{k=2}^{N} c_{k}(1-\beta)^{k-1}\left(\frac{1-\beta}{k-\frac{4}{3}}+\beta\right)$ & $c_{4}(1-\beta)^{3} \beta \lambda_{\mathrm{RD}}+\lambda_{\mathrm{RD}} \sum_{\substack{k=2 \\
k \neq 4}}^{N} c_{k}(1-\beta)^{k-1}\left(\frac{1-\beta}{k-4}+\beta\right)$ \\
\hline$B^{(S)}$ & 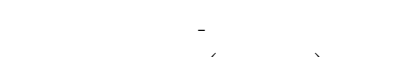 & $\frac{C_{4}(1-\beta)^{4} \lambda_{\mathrm{RD}}^{2}}{4}$ \\
\hline$E_{k}^{(S)}$ & $\frac{c_{k}(1-\beta)^{k} \lambda_{\mathrm{RD}}}{4}\left(1-\frac{k}{k-\frac{4}{3}}\right)$ & $\frac{c_{k}(1-\beta)^{k} \lambda_{\mathrm{RD}}}{4}\left(1-\frac{k}{k-\frac{4}{3}}\right), k \neq 4$ \\
\hline$a^{(S)}$ & $\frac{3}{2 \lambda_{\mathrm{RD}}}\left(\frac{2 A^{(S)} \lambda_{\mathrm{SD}}}{\frac{-2 \lambda_{\mathrm{RD}}}{3}+\lambda_{\mathrm{SD}}}\right)$ & $\frac{1}{2 \lambda_{\mathrm{RD}}}\left(\frac{2 A^{(S)} \lambda_{\mathrm{SD}}}{-2 \lambda_{\mathrm{RD}}+\lambda_{\mathrm{SD}}}-\frac{4 B^{(S)} \lambda_{S D}}{\left(-2 \lambda_{\mathrm{RD}}+\lambda_{\mathrm{SD}}\right)^{2}}\right)$ \\
\hline$b^{(S)}$ & - & $\frac{1}{4 \lambda_{\mathrm{RD}}^{2}}\left(\frac{4 B^{(S)} \lambda_{S D}}{-2 \lambda_{\mathrm{RD}}+\lambda_{S D}}\right)$ \\
\hline$e_{k}^{(S)}$ & $\frac{2}{\lambda_{\mathrm{RD}} k}\left(\frac{2 E_{k}^{(S)} \lambda_{\mathrm{SD}}}{-\frac{\lambda_{\mathrm{RD}}}{2}+\lambda_{\mathrm{SD}}}\right)$ & $\frac{2}{\lambda_{\mathrm{RD}} k}\left(\frac{2 E_{k}^{(S)} \lambda_{\mathrm{SD}}}{\frac{-\lambda_{\mathrm{RD}}}{2}+\lambda_{\mathrm{SD}}}\right)$ \\
\hline$f^{(S)}$ & $\frac{2 A^{(S)}}{\frac{2 \lambda_{\mathrm{RD}}}{3}-\lambda_{\mathrm{SD}}}+\sum_{k=2}^{N} \frac{2 E_{k}^{(S)}}{\left(\frac{\lambda_{\mathrm{RD}} D^{k}}{2}-\lambda_{\mathrm{SD}}\right)}+D^{(\mathrm{DF})}$ & $\frac{2 A^{(S)}}{2 \lambda_{\mathrm{RD}}-\lambda_{S \mathrm{D}}}+\frac{4 B^{(S)}}{\left(2 \lambda_{\mathrm{RD}}-\lambda_{\mathrm{SD}}\right)^{2}}+\underbrace{\sum_{k=2}^{N}}_{k \neq 4} \frac{2 E_{k}^{(S)}}{\frac{\lambda_{\mathrm{RD}}^{k}}{2}-\lambda_{\mathrm{SD}}}+D^{(\mathrm{DF})}$ \\
\hline
\end{tabular}

at the destination node for BF-AF (BA) in the second-time slot is obtained as [38]:

$$
\begin{aligned}
& y_{2 j}^{(\mathrm{BA})}=\sqrt{P_{S}} \sqrt{\frac{P_{S}}{2}} x_{j} \frac{\left|h_{\mathrm{RD}}^{(0)}\right|\left|h_{S_{j} R}^{(0)}\right|}{\sqrt{N_{o}+P_{S}\left|h_{S_{j} R}^{(0)}\right|^{2}}} \\
& +\sqrt{P_{S}} \sqrt{\frac{P_{S}}{2}} x_{j} \frac{\left|h_{\mathrm{RD}}^{(1)}\right|\left|h_{S_{j} R}^{(1)}\right|}{\sqrt{N_{o}+P_{S}\left|h_{S_{j} R}^{(1)}\right|^{2}}} \\
& +\sqrt{\frac{P_{S}}{2}} \frac{\left|h_{\mathrm{RD}}^{(0)}\right| n_{0 j}}{\sqrt{N_{o}+P_{S}\left|h_{S_{j} R}^{(0)}\right|^{2}}} \\
& +\sqrt{\frac{P_{S}}{2}} \frac{\left|h_{\mathrm{RD}}^{(1)}\right| n_{1 j}}{\sqrt{N_{o}+P_{S}\left|h_{S_{j} R}^{(1)}\right|^{2}}}+n_{2 j}^{(\mathrm{BA})}, \quad j=1,2 \text {, }
\end{aligned}
$$

where $n_{0 j}, n_{1 j}$ and $n_{2 j}^{(\mathrm{BA})}$ are the additive noise at the best and next-best relays in time slot $T_{1}$ and at the destination in time slot $T_{2}$, respectively.

The SNR $\gamma^{(\mathrm{BA})}$ at the destination node using MRC and using the best and next-best relays can be obtained as:

$$
\gamma^{(\mathrm{BA})}=\gamma_{S_{j} D}+\frac{\left(\sum_{i=0}^{1} \sqrt{\frac{\left|\gamma_{\mathrm{RD}}^{(i)}\right|\left|\gamma_{S_{j} R}^{(i)}\right|}{1+\left|\gamma_{S_{j} R}^{(i)}\right|}}\right)^{2}}{2\left(1+\sum_{i=0}^{1} \frac{\left|\gamma_{\mathrm{RD}}^{(i)}\right|}{2\left(1+\left|\gamma_{S_{j} R}^{(i)}\right|\right)}\right)},
$$

where $\gamma_{S_{j} R}^{(i)}=\gamma_{0}\left|h_{S_{j} R}^{(i)}\right|^{2}$, and $\gamma_{\mathrm{RD}}^{(i)}=\gamma_{0}\left|h_{\mathrm{RD}}^{(i)}\right|^{2} \cdot \gamma_{\mathrm{RD}}^{(i)}=$ $\gamma_{0}\left|h_{\mathrm{RD}}^{(i)}\right|^{2}$. It is also difficult here to compute analytical expressions for the PDF and the MGF of $\gamma^{(\mathrm{BA})}$ at the destination node. Therefore, an upper bound for the SNR $\gamma^{(\mathrm{BA})}$ is given as [38]:

$$
\begin{aligned}
\gamma^{(\mathrm{BA})} & \leq \gamma_{\mathrm{O} p}^{(\mathrm{BA})}=\gamma_{S_{j} D}+\frac{\gamma_{S_{j} R}^{(0)} \gamma_{\mathrm{RD}}^{(0)}}{1+\gamma_{S_{j} R}^{(0)}+\gamma_{\mathrm{RD}}^{(0)}}+\frac{\gamma_{S_{j} R}^{(1)} \gamma_{\mathrm{RD}}^{(1)}}{1+\gamma_{S_{j} R}^{(1)}+\gamma_{\mathrm{RD}}^{(1)}} \\
& \leq \gamma_{S_{j} D}+\gamma_{b_{0}}^{(\mathrm{BA})}+\gamma_{b_{1}}^{(\mathrm{BA})}
\end{aligned}
$$

where $\gamma_{O p}^{(\mathrm{BA})}$ is defined as the maximum received SNR using optimum power assignment for the best, and the next-best ordered relays under the constraint $P_{R}^{(0)}+$ $P_{R}^{(1)}=P_{S} \cdot \gamma_{b_{0}}^{(\mathrm{BA})}=\max _{l}\left(\min \left(\gamma_{S_{j} R_{l}}, \gamma_{R_{l} D}\right)\right)$, and $\gamma_{b_{1}}^{(\mathrm{BA})}=$ $\max _{l, l \neq b_{0}}\left(\min \left(\gamma_{S_{j} R_{l}}, \gamma_{R_{l} D}\right)\right)$. Hence, the lower bound of the $\mathrm{BEP}^{(\mathrm{BA})}$ can be found using (30) by replacing $\lambda_{\mathrm{eq}}=$ $2 \lambda_{\mathrm{SR}}+\lambda_{\mathrm{RD}}$ with $\lambda_{\text {eq }}=\lambda_{\mathrm{SR}}+\lambda_{\mathrm{RD}}$.

It is worth noting that the weights for BF AF and DF are chosen as $w_{1}=1$ and $w_{2}=1$ for the following reasons. First, the weights $w_{1}$ and $w_{2}$ can be considered as power adjustment factors, and in this study optimal power adjustment is not considered, only equal power assignment is investigated for all the scenarios. Second, the weights are not similar to the weights given by $[16,38]$ which were derived for one user scenario. The weights for this problem need to be selected to maximize the minimum received SNR of the two users under individual relay power constraint and user sum power constraint. Finally, if power adjustment is to be used, there is no benefit from relay ordering, relay ordering in this article is based on relays' equal power transmission.

\section{Numerical results and discussion}

In this section, we provide the results of the BEP performance for sharing the two ordered best relays, and compare it with the performance of the best ordered and the next-best ordered relays using Monte Carlo simulations 


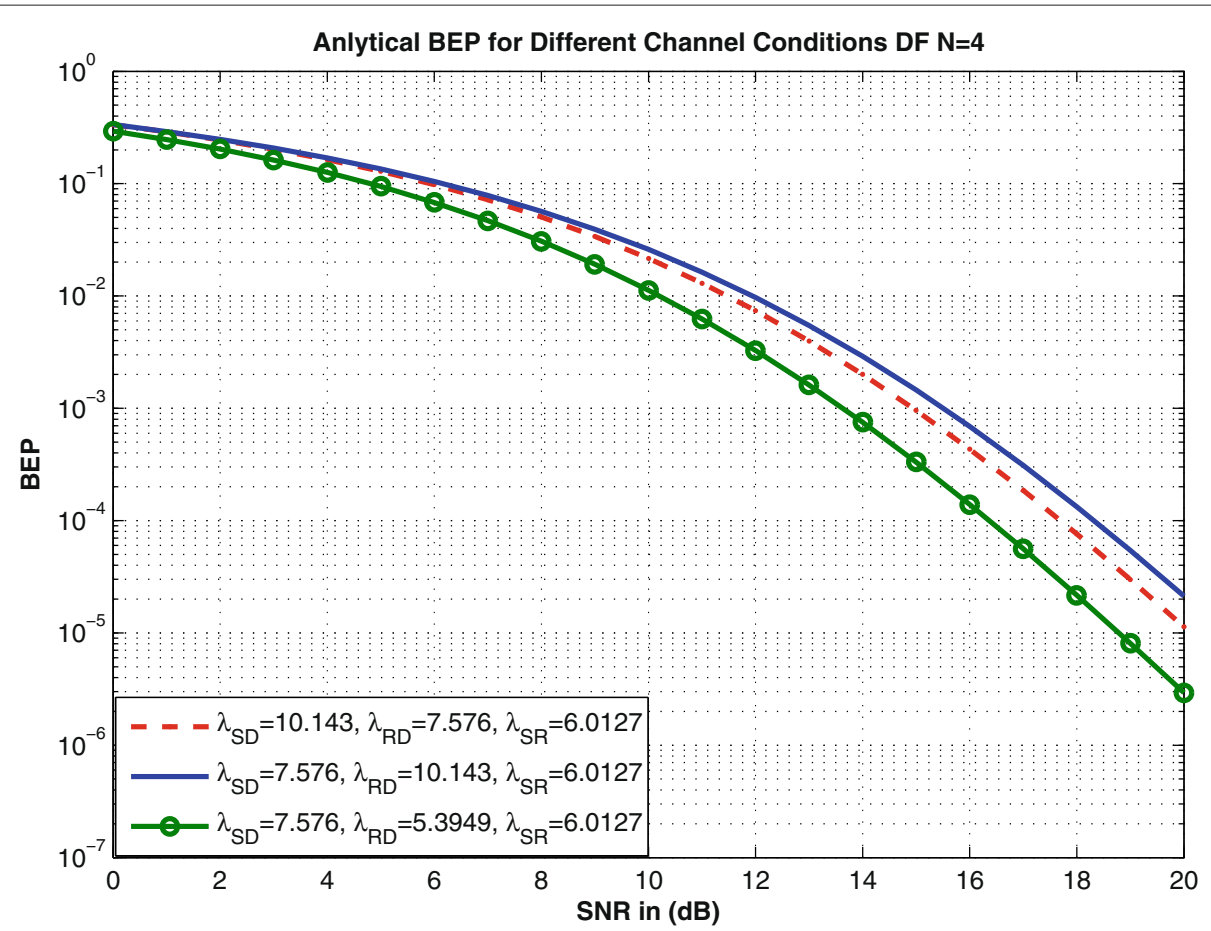

Figure 2 The BEP performance for the sharing DF scenario for different channels' conditions using analytical formula.

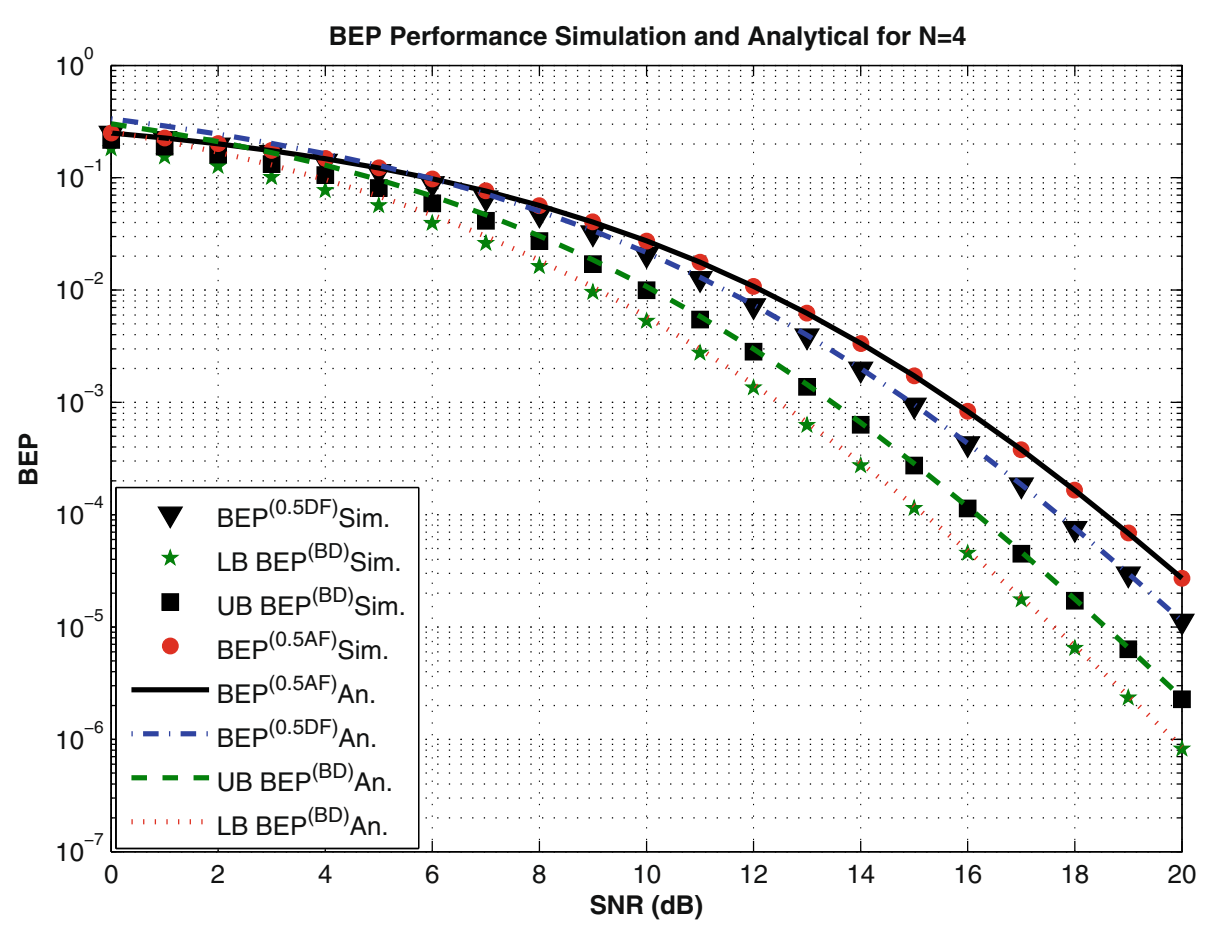

Figure 3 Comparison of the BEP performance using derived formulas and simulations for the sharing of the best ordered relays $R_{b 0}, R_{b 1}$. 


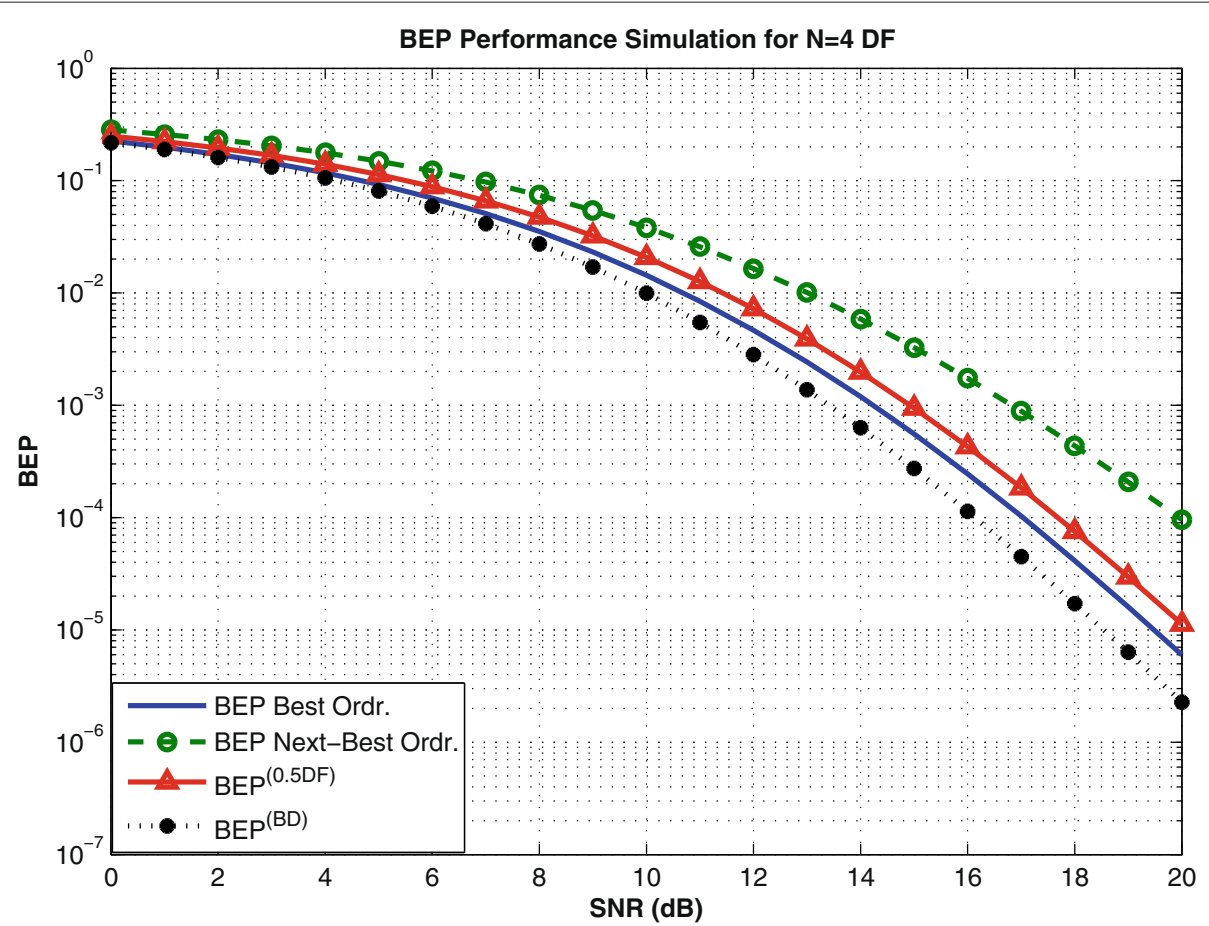

Figure 4 The BEP performance for the sharing DF scenario using simulation.

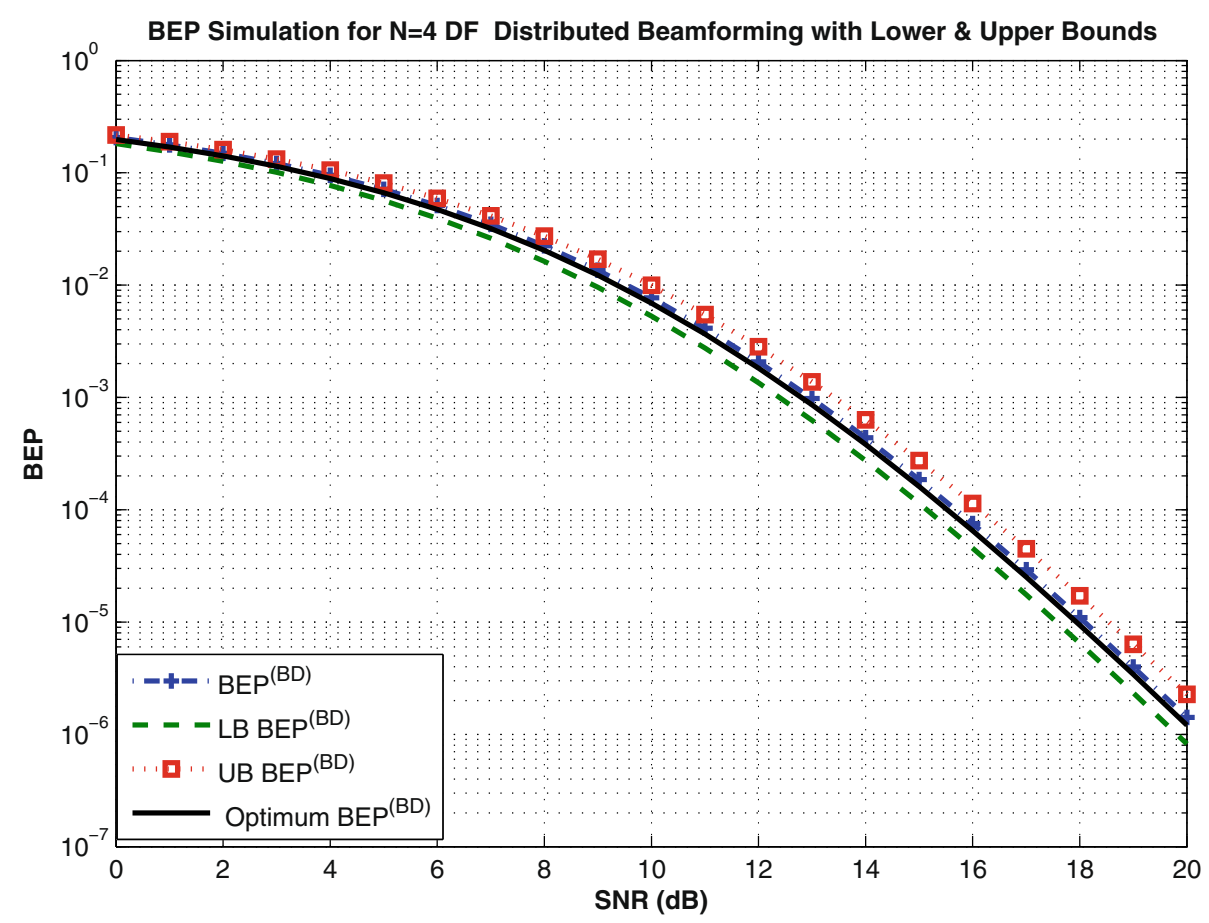

Figure 5 The BEP performance for DF distributed BF scenario using simulation. 


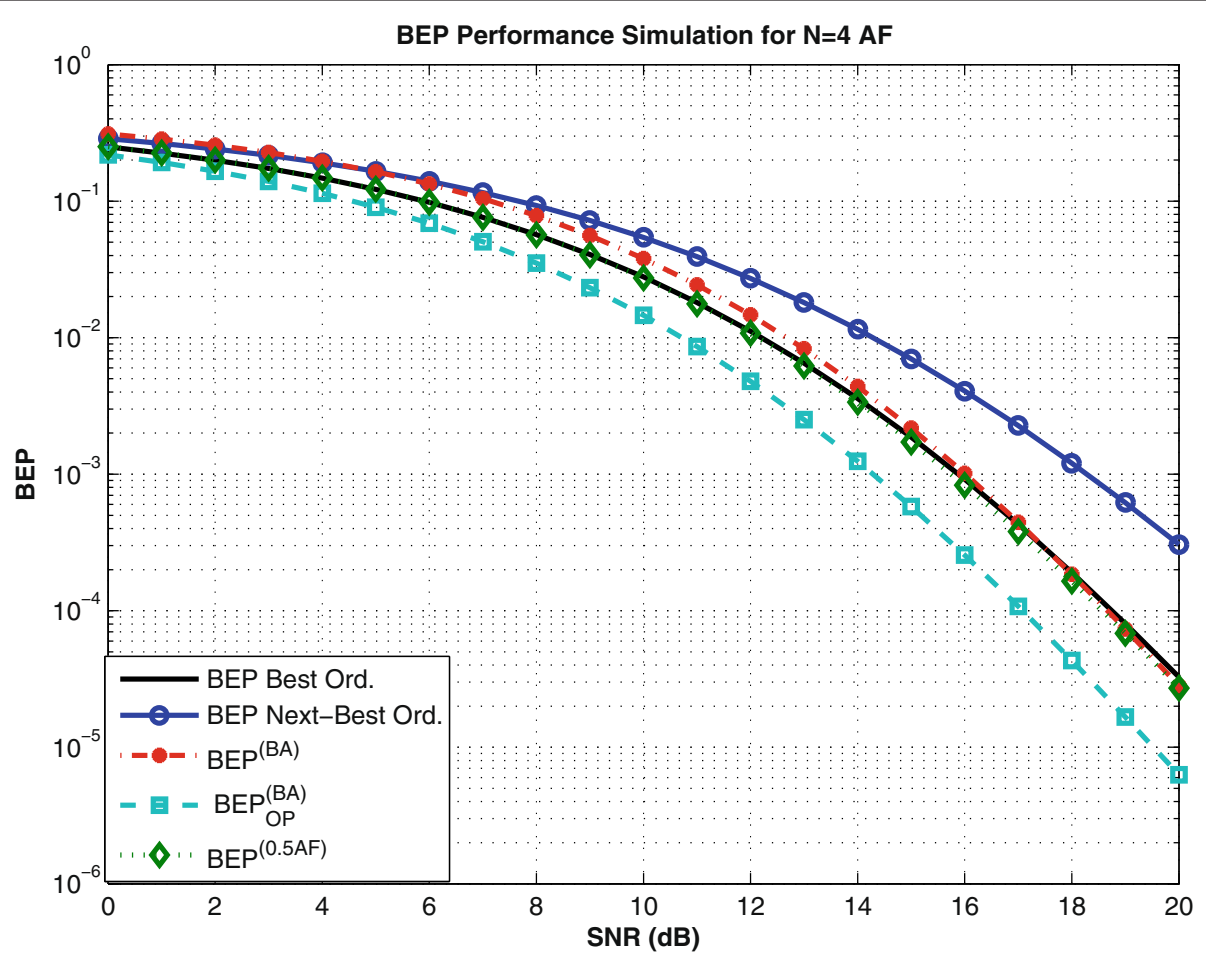

Figure 6 The BEP performance for AF sharing scenario using simulation.

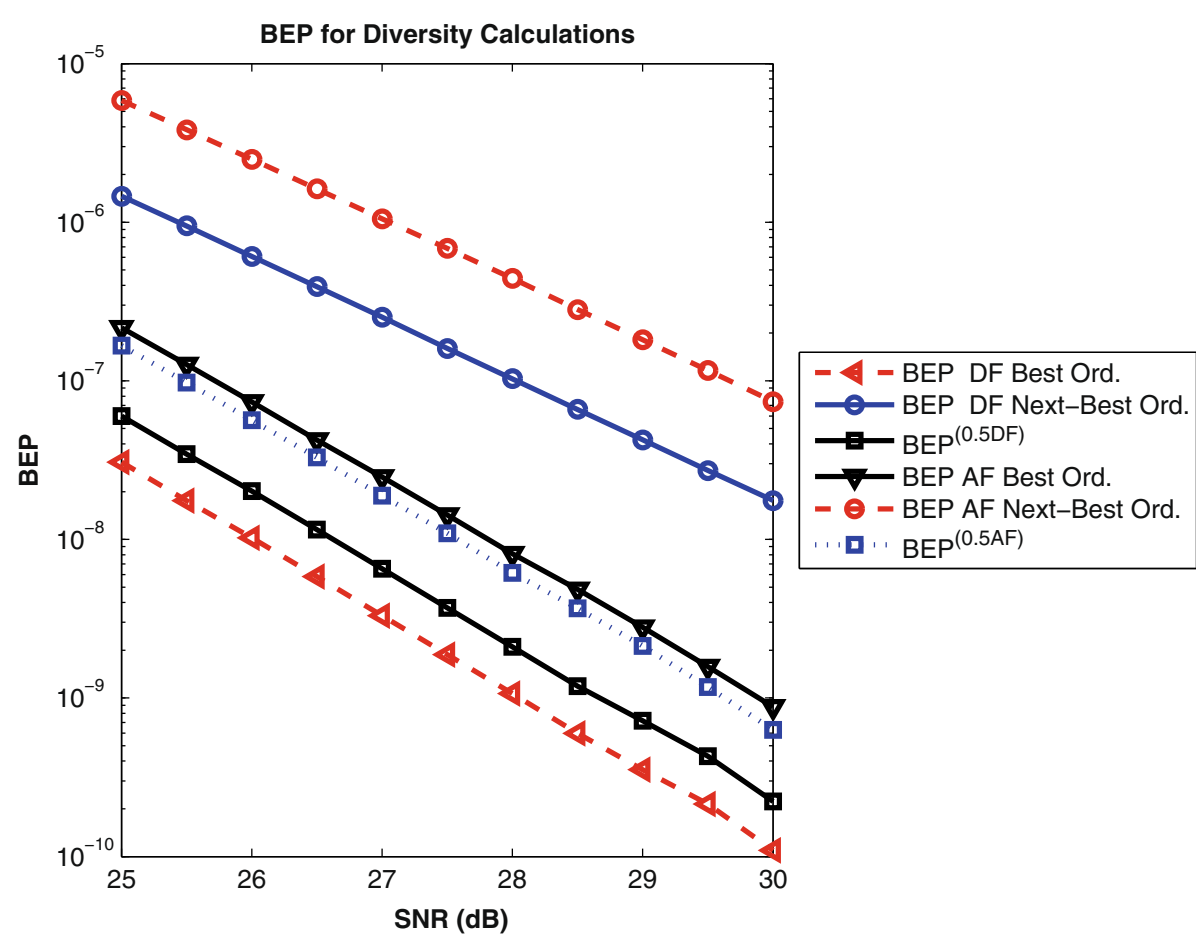

Figure 7 The BEP performance for diversity calculations for the orthogonal sharing AF and DF schemes using simulations. 
for $N=4$. In all simulations and analytical expressions $\lambda_{\mathrm{SD}}=10, \lambda_{\mathrm{RD}}=7.5$ and $\lambda_{\mathrm{SR}}=6.0$ are used to simulate a better average source-relay and relay-destination channel links than a source-destination link except in Figure 2, where three different conditions are considered as explained later.

In Figure 3, the analytical BEP performance is compared to the simulated BEP performance for both DF and AF schemes in two time slots scenario and three-time slots scenario. As clear from this figure, the BEP curves using simulations exactly coincide with those obtained analytically for orthogonal DF (22), the upper bound of the distributed BF-DF (39), the lower bound of BF-DF (40), and the lower bound of AF (30). An SNR gain of $0.85 \mathrm{~dB}$ for the case of DF cooperation scheme is observed compared to AF cooperation scheme measured at $\mathrm{BEP}=10^{-4}$.

In Figure 4 simulation results of the BEP performance for sharing the two ordered best relays in DF cooperation scheme are shown and compared with the BEP performance of the best and the next-best ordered relays. As shown in this figure, sharing the two best ordered relays outperforms the next-best relay by $2.2671 \mathrm{~dB}$ at $\mathrm{BEP}=10^{-4}$. The best ordered relay $\mathrm{BEP}$ performance outperforms the sharing BEP performance by $0.7099 d B$ at $\mathrm{BEP}=10^{-4}$. We also compare the BEP performance of the three time slots DF with the BEP performance of the two time slots BF-DF scenario. An SNR gain of $0.8244 \mathrm{~dB}$ for BF-DF is observed compared to DF best relay at $\mathrm{BEP}=10^{-4}$.

For distributed BF DF cooperation schemes the exact BEP performance is obtained and compared with the BEP performance upper bound, BEP performance lower bound and BEP performance with optimal power allocation strategy. The simulation results are shown in Figure 5. As clear from this figure, the upper and lower bounds are pretty tight. The SNR gain of the BEP performance with optimal power strategy is very marginal. The SNR gain of the optimal power strategy is $0.1558 \mathrm{~dB}$ compared to equally sharing the best and next best ordered relays at $\mathrm{BEP}=10^{-4}$.

In Figure 6, the BEP performance of sharing the two ordered best relays in AF scheme is obtained and compared to the BEP performance of the best and nextbest ordered relays. The BEP performance of the sharing scenario outperforms the best relay by $0.1829 \mathrm{~dB}$ at $\mathrm{BEP}=10^{-4}$. In addition, we simulate the BEP performance of BA, which approaches that of the best ordered relay at high SNR. The BEP performance of BF-AF using optimal power allocation under total power constraint is also shown. The SNR gain of the BEP performance with optimal power allocation is $0.7 \mathrm{~dB}$ compared to sharing the best and next best ordered relays at $\mathrm{BEP}=10^{-4}$.
Figure 2 shows results of the analytical BEP performance for three channel conditions for DF with sharing the best and next best ordered relays. In setup $1, \lambda_{\mathrm{SD}}=$ 10.143, $\lambda_{\mathrm{RD}}=7.576$ and $\lambda_{\mathrm{SR}}=6.0127$. In setup 2 , $\lambda_{\mathrm{SD}}=7.576, \lambda_{\mathrm{RD}}=10.143$ and $\lambda_{\mathrm{SR}}=6.0127$. Finally, in setup $3, \lambda_{\mathrm{SD}}=7.576, \lambda_{\mathrm{RD}}=5.394$ and $\lambda_{\mathrm{SR}}=6.0127$. The BEP performance of channel setup 3 outperforms the BEP performance of the other setups. An SNR gain of $1.3022 \mathrm{~dB}$ and $1.9301 \mathrm{~dB}$ at $\mathrm{BEP}=10^{-4}$ is observed compared to setup 2 and setup 1 respectively. It is well observed that, a better BEP performance is achieved when the source-relay and the relay-destination links are in better channel conditions than source-destination link.

In Figure 7, the BEP performance is shown at a high SNR range from $25 \mathrm{~dB}$ to $30 \mathrm{~dB}$ for sharing the best and nextbest relays, the best relay alone, and the next-best relay alone for both AF and DF schemes. In all schemes, analytically, the diversity order is 5, except for the next best relay where the diversity order is 4 . The diversity order is calculated using simulations as shown in Table 8. As shown in this table, the diversity order of sharing the two ordered best relays for both DF and AF schemes is the same as the diversity order of the best relay. The slope of the BEP performance of sharing the best and next best relays is approximately the same as the slope of the BEP performance of the best relay. Two SNR values are considered for comparison purpose $\left(S N R_{1}=27 \mathrm{~dB}\right.$ and $\left.S N R_{2}=30 \mathrm{~dB}\right)$. The diversity order is computed in the last column of Table 8 .

\section{Conclusions}

In this article, relay selection for cooperative networks is investigated. Assuming two users scenario, and $N$ relays are available, BEP and outage probability are computed for the case of sharing the two ordered best relays for AF and DF relaying schemes. Analytical expressions for the BEP and the outage probability are derived for the different scenarios. Simulation results validate the analytical expressions of the BEP performance. The BEP performance of the proposed schemes were also compared with the BEP performances of the best and next-best relays. In DF scheme, the BEP performance of the best relay

\section{Table 8 Diversity calculations}

\begin{tabular}{llll}
\hline Scheme & BEP $_{\mathbf{1}}$ & BEP $_{\mathbf{2}}$ & Diversity order \\
\hline Best AF & $2.4777 \times 10^{-8}$ & $8.7832 \times 10^{-10}$ & 4.8181 \\
Next-best AF & $1.0507 \times 10^{-6}$ & $7.4121 \times 10^{-8}$ & 3.8253 \\
Sharing AF & $1.8862 \times 10^{-8}$ & $6.2973 \times 10^{-10}$ & $\mathbf{4 . 9 0 4 6}$ \\
Best DF & $3.3840 \times 10^{-9}$ & $1.0909 \times 10^{-10}$ & 4.9551 \\
Next-best DF & $2.5017 \times 10^{-7}$ & $1.6573 \times 10^{-8}$ & 3.9160 \\
Sharing DF & $6.6892 \times 10^{-9}$ & $2.0673 \times 10^{-10}$ & $\mathbf{5 . 0 1 6 0}$ \\
\hline
\end{tabular}


outperforms the BEP performance of the sharing scenario. But, the BEP performance of BF-DF scenario outperforms the BEP performance of the ordered best relay. Furthermore, the BEP performance of equally sharing the two best ordered relays for AF outperforms the BEP performance of the ordered best relay. Efficient channel utilization is achieved by using STBC and BF.

\section{Endnotes}

${ }^{\text {a }}$ The decoding set $C$ is a subset of the $N$ available relays. If a relay is not in the decoding set, the end-to-end SNR value is set to zero.

${ }^{\mathrm{b}} \mathrm{A}$ function $a(x)$ is written as $O(x)$ if $\lim _{x \rightarrow 0} \frac{a(x)}{x}=0$.

\section{Competing interests}

The authors declare that they have no competing interests.

Received: 1 November 2011 Accepted: 18 June 2012

Published: 19 July 2012

\section{References}

1. A Sendonaris, E Erkip, B Aazhang, User cooperation diversity: part I system description. IEEE Trans. Commun. 51(11), 1939-1948 (2003)

2. A Sendonaris, E Erkip, B Aazhang, User cooperation diversity: part II implementation aspects and performance analysis. IEEE Trans. Commun. 51(11), 1939-1948 (2003)

3. J Laneman, D Tse, G Wornell, Cooperative diversity in wireless networks: efficient protocols and outage behavior. IEEE Trans. Inf. Theory. 50(12) 3062-3080 (2004)

4. J Laneman, Cooperative diversity in wireless networks: algorithms and architectures. Ph.d. thesis, Massachusetts Institute of Technology, Cambridge, MA, Aug 2002

5. J Hu, X Chen, Performance of decode-and-forward cooperative communications with channel estimation errors over Rayleigh fading channels. in 2nd International Conference on Future Computer and Communication (ICFCC), vol. 1 (2010), vol. 1 pp. 164-167

6. J Laneman, Network coding gain of cooperative diversity. in Military Communications Conference, MILCOM 2004, vol. 1, pp. 106-112

7. $\mathrm{P}$ Anghel, M Kaveh, Exact symbol error probability of a cooperative network in a Rayleigh-fading environment. IEEE Trans. Wirel. Commun. 3(5), 1416-1421 (2004)

8. A Ribeiro, X Cai, G Giannakis, Symbol error probabilities for general cooperative links. IEEE Trans. Wirel. Commun. 4(3), 1264-1273 (2005)

9. N Vien, H Nguyen, T Le-Ngoc, Diversity analysis of smart relaying. IEEE Trans. Veh. Technol. 58(6), 2849-2862 (2009)

10. TWang, A Cano, G Giannakis, JN Laneman, High-performance cooperative demodulation with decode-and-forward relays. IEEE Trans. Commun. 55(7), 1427-1438 (2007)

11. A Ribeiro, X Cai, G Giannakis, Opportunistic multipath for bandwidth-efficient cooperative multiple access. IEEE Trans. Wirel. Commun. 5(9), 2321-2327 (2006)

12. M Torabi1, W Ajib, D Haccoun, Performance analysis of amplify-andforward cooperative networks with relay selection over Rayleigh fading channels. in IEEE 69th Vehicular Technology Conference (2009), pp. 1-5

13. Y Zhao, R Adve, T Lim, Symbol error rate of selection amplify-and-forward relay systems. IEEE Commun. Lett. 10(11), 757-759 (2006)

14. S Ikki, M Ahmed, Performance of multiple-relay cooperative diversity systems with best relay selection over Rayleigh fading channels. EURASIP J. Adv. Signal Process. vol.1. 145, 1-7 (2008)

15. A Bletsas, H Shin, M Win, Cooperative communications with outage-optimal opportunistic relaying. IEEE Trans. Wirel. Commun. 6(9), 3450-3460 (2007)

16. $Y$ Jing, $H$ Jafarkhani, Single and multiple relay selection schemes and their achievable diversity orders. IEEE Trans. Wirel. Commun. 8(3), 1414-1423 (2009)
17. S Ikki, M Ahmed, On the performance of cooperative-diversity networks with the Nth best-relay selection scheme. IEEE Trans. Commun. 58(11) 3062-3069 (2010)

18. E Beres, R Adve, Selection cooperation in multi-source cooperative networks. IEEE Trans. Wirel. Commun. 7(1), 118-127 (2008)

19. W Guo, J Liu, L Zheng, Y Liu, G Zhang, Performance analysis of a selection cooperation scheme in multi-source multi-relay networks. in International Conference on Wireless Communications and Signal Processing (WCSP) (2010), pp. 1-6

20. X Zhang, W Wang, X Ji, Multiuser diversity in multiuser two-hop cooperative relay wireless networks: system model and performance analysis. IEEE Trans. Veh. Technol. 58(2), 1031-1036 (2009)

21. S Ikki, M Ahmed, On the performance of adaptive decode-and-forward cooperative diversity with the Nth best-relay selection scheme. in IEEE Global Telecommunications Conference, GLOBECOM 2009 (2009), pp. 1-6

22. S Ikki, M Ahmed, On the performance of amplify-and-forward cooperative diversity with the Nth best-relay selection scheme. in IEEE International Conference on Communications, ICC'09 (2009), pp. 1-6

23. C Yang, S Zhao, W Wang, M Peng, Performance of decode-and-forward opportunistic cooperation with the Nth best relay selected. in Proceedings of the 6th International Wireless Communications and Mobile Computing Conference IWCMC, (2010), pp. 1253-1257

24. K Woradit, T Quek, W Suwansantisuk, H Wymeersch, L Wuttisittikulkij, M Win, Outage behavior of selective relaying schemes. IEEE Trans. Wirel. Commun. 8(8), 3890-3895 (2009)

25. S Ikki, M Ahmed, Performance analysis of adaptive decode-and-forward cooperative diversity networks with best-relay selection. IEEE Trans. Commun. 58(1), 68-72 (2010)

26. N Beaulieu, J Hu, A closed-form expression for the outage probability of decode-and-forward relaying in dissimilar Rayleigh fading channels. IEEE Commun. Lett. 10(12), 813-815 (2006)

27. H David, H Nagaraja, Order Statistics, 3rd edn. (Wiley, New York, 2003)

28. O Ibe, Fundamentals of Applied Probability and Random Processes (Elsevier Academic Press, Amsterdam, 2005)

29. A Papoulis, Probability, Random Variables, and Stochastic Processes, 3rd edn. (McGraw-Hill, New York, 1991)

30. M Simon, M Alouini, Digital Communication Over Fading Channels (Wiley, New York, 2000)

31. Z Wang, G Giannakis, A simple and general parameterization quantifying performance in fading channels. IEEE Trans. Commun. 51(8), 1389-1398 (2003)

32. A Annamalai, G Deora, C Tellambura, Analysis of generalized selection diversity systems in wireless channels. IEEE Trans. Veh. Technol. 55(6), 1765-1775 (2006)

33. Q Liu, X Ma, G Zhou, A general diversity gain function and its application in amplify-and-forward cooperative networks. IEEE Trans. Signal Process. 59(2), 859-863 (2011)

34. M Hasna, M Alouini, End-to-end performance of transmission systems with relays over Rayleigh-fading channels. IEEE Trans. Wirel. Commun. 2(6), 1126-1131 (2003)

35. S Alamouti, A simple transmit diversity technique for wireless communications. IEEE J Sel. Areas Commun. 16(8), 1451-1458 (1998)

36. Z Bai, D Yuan, K Kwak, Performance evaluation of STBC based cooperative systems over slow Rayleigh fading channel. Comput. Commun. 31(17), 4206-4211 (2008). http://www.sciencedirect.com/science/article/B6TYP4TF7CB9-3/2/360a5d1dfe9a667ca91e1a2295786f17

37. $Y$ Jing, B Hassibi, Distributed space-time coding in wireless relay networks. IEEE Trans. Wirel. Commun. 5(12), 3524-3536 (2006)

38. $\mathrm{P}$ Larsson, $\mathrm{H}$ Rong, Large-scale cooperative relay network with optima coherent combining under aggregate relay power constraints. in The Future Telecommunications Conference, Beijing FTC2003 166170, (2003)

doi:10.1186/1687-1499-2012-224

Cite this article as: Al-Tous and Barhumi: Performance analysis of relay selection in cooperative networks over Rayleigh flat fading channels. EURASIP Journal on Wireless Communications and Networking 2012 2012:224. 